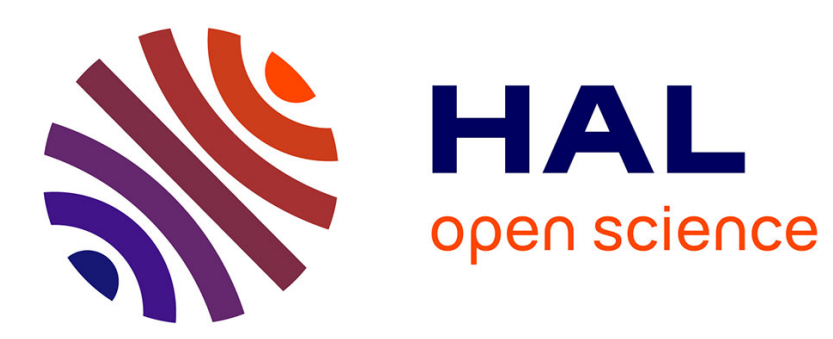

\title{
Turbulent drag induced by low surface roughness at transonic speeds: Experimental/numerical comparisons
}

\author{
David Hue, Pascal Molton
}

\section{To cite this version:}

David Hue, Pascal Molton. Turbulent drag induced by low surface roughness at transonic speeds: Experimental/numerical comparisons. Physics of Fluids, 2020, 32 (4), pp.045108-1 - 045108-18. 10.1063/1.5143068 . hal-02906130

\section{HAL Id: hal-02906130 \\ https://hal.science/hal-02906130}

Submitted on 24 Jul 2020

HAL is a multi-disciplinary open access archive for the deposit and dissemination of scientific research documents, whether they are published or not. The documents may come from teaching and research institutions in France or abroad, or from public or private research centers.
L'archive ouverte pluridisciplinaire HAL, est destinée au dépôt et à la diffusion de documents scientifiques de niveau recherche, publiés ou non, émanant des établissements d'enseignement et de recherche français ou étrangers, des laboratoires publics ou privés. 


\title{
Turbulent drag induced by low surface roughness at transonic speeds: experimental / numerical comparisons
}

\author{
David Hue* ${ }^{1}$, Pascal Molton ${ }^{2}$, \\ ONERA - The French Aerospace Lab, 92190 Meudon, France
}

The paper gives the main results of an experimental test campaign aimed at quantifying the effects of very low roughness levels on the flat plate turbulent drag in transonic conditions. This work, in a field not completely understood yet, the one of the transitionally rough regime, has revealed its importance by showing that some surfaces considered as hydrodynamically smooth in past studies might not have been. That issue can be strongly critical in the delicate exercise of experimental / numerical comparisons, and more specifically, when it comes to absolute drag predictions, if the fluid dynamics computations use only, as they almost always do, infinitely smooth surfaces. The experiments carried out in the S8Ch wind tunnel have involved high-level measurement techniques, such as micro-drag evaluation with a three-component balance and near-wall laser Doppler velocimetry, for Mach numbers from 0.55 to 0.8 and maximum Reynolds numbers based on the sample length and boundary layer thickness of about 2.6 million and 0.13 million respectively, the greatest friction Reynolds number being close to 5,000. A dozen surface samples were tested, with average roughness values from less than $0.25 \mu \mathrm{m}$ (mirror-polished aluminum) to more than $10 \mu \mathrm{m}$ (commercial sandpapers), and in between standard-machined or painted samples (including pressure sensitive paintings). A pragmatic computational fluid dynamics study reproducing this test campaign was completed. It is based on the well-known equivalent sand grain roughness height approach and also on the Musker correlation, which was adapted. The results and validity of such Reynolds-averaged Navier-Stokes simulations in that particular regime are discussed. Anyway, both experimental and numerical outcomes of this

\footnotetext{
${ }^{1}$ Research Engineer, Aerodynamics, Aeroelasticity, Acoustics Department, david.hue@onera.fr.

${ }^{2}$ Research Engineer, Aerodynamics, Aeroelasticity, Acoustics Department, pascal.molton@onera.fr
} 
work are in agreement to indicate that drag can be significantly impacted even for roughness Reynolds numbers potentially below the usual threshold values often considered in the engineering world (i.e. about 3.5 to 5). And the cross-analysis of surface drag production and roughness characteristics has allowed the decisive role played by the rms (or average) roughness height, the skewness and kurtosis coefficients and more especially the slope parameter to be confirmed.

\section{Introduction}

VEN if this study deals with the specific topic of very low surface roughness at transonic speeds, a domain

$\mathrm{E}_{\text {in }}$ n which the recent literature is not so abundant, at least not abundant enough to ensure that all of the key aspects are perfectly understood, many researchers have already published excellent historical introductions or reviews concerning the turbulent flows over rough walls, for instance Jiménez [1] or Flack and Schultz [2]. As a consequence, the objective in this paragraph will only be to give a brief general overview and to emphasize the particular points that will be useful to situate the present work.

Among the first widely spread studies focused on the effects of roughness on wall fluid flows is of course the one of Nikuradse [3] in 1933. It consisted in series of experiments measuring the hydraulic losses encountered with different sizes of sand grains in pipes, which gave birth to the famous notion of equivalent sand grain roughness height $k_{s}$ that will be discussed later. Then, closely after him, Colebrook [4] in 1939, and Moody [5] in 1944 who used the progress made by the latter to elaborate the unmissable diagram giving the pressure drop coefficient versus the Reynolds number and pipe roughness. Diagram which, among others, has the advantage to highlight the existence of three different regimes: hydrodynamically smooth, fully rough (in which the roughness is so important that the hydraulic loss coefficient does not depend on the Reynolds number anymore), and between them, the transitionally rough regime, where the friction is equivalent or even dominates the pressure forces. Despite its confusing name, this regime has nothing to do with the laminar to turbulent transition: it only corresponds to the situation in which the couple "surface + flow" is neither in the hydrodynamically smooth regime nor in the fully rough one.

What is at stake, and it has not changed since the pioneering days, is by knowing the roughness of a surface and those who tried will admit that having the roughness of a non-academic surface completely characterized is quite 
a complex issue in itself - to be able to predict its drag for a given set of flow conditions. And in the case of very small roughness imperfections, belonging to the transitionally rough regime and therefore associated with low roughness Reynolds number $k_{s}{ }^{+}=\left(U_{t} / v\right)^{*} k_{s}$, as it can be defined $-k_{s}{ }^{+}$being then a dimensionless parameter which measures the effect of roughness on the buffer layer - the literature readily concedes that many unresolved questions remain.

One of them is to determine from which value of $k_{s}^{+}$, or which roughness height for a given flow, a surface sample will produce a drag distinctively greater than the one of the surface considered as (sometimes wrongly) perfectly smooth. The threshold values of $k_{s}{ }^{+}$taken into account in the practical engineering field to estimate if a surface is hydrodynamically smooth are usually in the range of 3.5 to 5 (Nikuradse-type). And non-experts often tend to think that if $k_{s}^{+}$is just a bit above these values, then the drag should not be largely different from the one of the reference smooth sample or model. Well, it seems that these seductive ideas have hidden a reality in which a lot of different situations can occur: the fact that the surfaces do not follow a universal behavior in the transitionally rough regime now appears to be a shared opinion. And quite recently, several researchers, including Flack and Schultz [6] in 2012, have shown that roughness Reynolds numbers ${k_{s}}^{+}$as low as 2 or even lower can be enough to measure a non-negligible force increase compared to the drag of the smooth surface. Other scientists, like Bradshaw [7] in 2000, suggest that there is no justification for such threshold values concerning the effect of roughness and so that it should have an impact as soon as $k_{s}^{+}$is no longer zero.

Another crucial issue, partly overlapping the former one, is the form itself of the roughness function in the transitionally rough regime. This function models the downward shift in the boundary layer log law due to the wall roughness. There are two main branches, whose names are based on the ones of the precursors: the Nikuradse and the Colebrook functions, as illustrated in Fig. 1. As evocated, the Nikuradse-type clearly exhibits a hydrodynamically smooth regime for $k_{s}^{+}<\sim 5$ and then an abrupt departure into the transitionally rough one whereas the form of the Colebrook-type shows a soft growth starting directly from $k_{s}^{+}=0$, which is consistent with the position of Bradshaw. And according to the flow conditions, according to the roughness characteristics of the surface being considered, what is observed in experiments or in computations is either rather close to one behavior or rather close to the other, and sometimes perfectly between them. As a consequence, the question of knowing a priori which function should be applied to a given couple "surface + flow" is still open. Some recent numerical works, using large eddy (LES) or direct (DNS) simulations, for example the ones of Yuan and Piomelli [8] in 2014 and of Thakkar, 
Busse, and Sandham [9,10] in 2017 and 2018 (with maximum friction Reynolds numbers below 1,000 which is relatively moderate compared to the ones that can be reached in transonic wind tunnels), even propose new formulations for the roughness function by dissociating two different transitionally rough regimes: a lower one and an upper one.

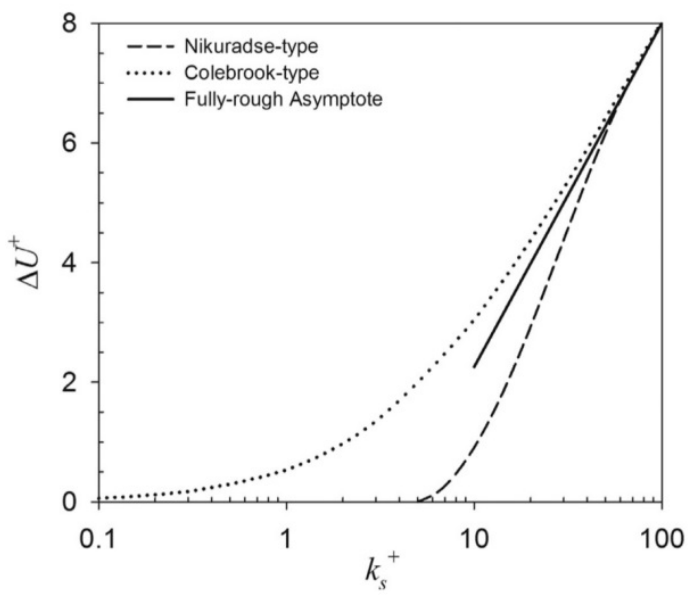

Fig. 1 The roughness function $\Delta U^{+}$versus $k_{s}^{+}$for Nikuradse-type and Colebrook-type roughness.

In parallel with these studies aiming at achieving a better modelling of the phenomenon, the approach already mentioned and known as the equivalent sand grain roughness height has been widely used and enriched, from the sixties till nowadays, especially because of its easy implementation in computational fluid dynamics (CFD) codes from which the drag value taking the roughness into account is then extracted ( $C D$ is obtained with $\Delta U^{+}$that is given via $k_{s}{ }^{+}$calculated itself with $k_{s}$ ). And one of the greatest objectives has been to define empirical correlations allowing $k_{s}$ to be determined by using some of the surface roughness characteristics as inputs. For instance, the correlations of Bammert and Sandstede in 1976, $k_{s}=2.2 R a^{0.877}$, of Schaffler in 1980, $k_{s}=8.9 R a$, can be mentioned as well as the one of Musker [11] which already in 1981 implied more advanced features: $k_{s}=R q(1+a * S p)\left(1+b * S k^{*} K u\right)$, where $R a$ an $R q$ are the average and rms roughness heights, $S k$ and $K u$ are the skewness and kurtosis coefficients and $S p$ the slope parameter. In 2010, Bons [12] published a review that gathers a large number of these relations. More recently and proving the ongoing interest for this matter, the article Toward a Universal Roughness Correlation by Forooghi et al. [13] in 2017, which presents the analysis of DNS results, is to be noticed. And here again, the work of Flack and Schultz [14] in 2009 who emphasized the importance of the effective slope parameter - highlighted by Napoli [15] - in the roughness effects, related to the notion of "waviness regime”, while limiting to $R q$ and $S k$ the variables 
they use in their own correlations [2,16] of 2014 and 2016 as for example: $k_{s}=4.43 R q(1+S k)^{1.37}$. It should be noted, as will be addressed in a further section, that all these formulas giving $k_{s}$ only from geometrical surface characteristics are not supposed to be suitable for the transitionally rough regime since in this case $k_{s}$ might depend on the surface and Reynolds number, not just on the surface like it should in the fully rough regime.

In 2018, Barros, Flack and Schultz [17] came back on the influence of the slope parameter, in particular for surfaces with low slopes, and obtained behaviors quite close to the Colebrook-type roughness function, as shown in the third figure of their paper, especially as the slope value decreases, implying significant levels of $\Delta U^{+}$compared to the Nikuradse-type behavior even for very low $k_{s}{ }^{+}$values (definitely below 3.5), which seems to be in good agreement with what was observed in the present study.

At first, this article will describe the experimental set-up used in the ONERA S8Ch wind tunnel $\left(0.12 \times 0.1 \mathrm{~m}^{2}\right.$ test section) to quantify the zero-pressure-gradient turbulent drag of a dozen $0.2 \mathrm{~m}$ long flat plate samples exhibiting different roughness levels. Eight of them will be presented in detail here, their roughness features and measured profiles will be given. The range of $R a$ investigated varies from about $0.25 \mu \mathrm{m}$ for the mirror-polished aluminum sample to more than $10 \mu \mathrm{m}$ for the harshest commercial sandpaper, and the slope parameter $S p$ ranging from about 0.025 to 0.5 . The set of samples includes standard-machined or painted flat plates. Each of them was tested for transonic Mach numbers from 0.55 to 0.8 , it corresponds to maximum Reynolds numbers based on the sample length and on the boundary layer thickness of 2.6 million and 0.13 million, the highest friction Reynolds number reaching about 5,000. The procedures of data acquisition with the measurement tools, such as the micro-force KISTLER balance and the near-wall laser instruments, will be described as well.

Then, the focus will be on the characteristics of the Reynolds-averaged Navier-Stokes (RANS) simulations reproducing the S8Ch experiments. The approach in this part of the work is clearly pragmatic since it is based on the equivalent sand grain height method, whose limitations have been evocated above. A structured grid and the inhouse aerodynamic solver elsA ( $2^{\text {nd }}$ order accuracy) were used, as well as a correlation adapted from the one of Musker to determine the necessary $k_{s T r a n s}$ values.

Before arriving to the sections that show the outcomes and to give some further insight on the variable values allowing this study to be situated in the literature panorama, a part will be dedicated to the roughness Reynolds number $k^{+}$and $R a / \delta$ ranges which extend, considering the very low roughness levels tackled in this project, from below 1 to about 100 and from $0.002 \%$ to $0.1 \%$ respectively. 
Finally, the experimental and numerical results will be presented and compared, firstly with the global forces and then with the local aspects. The evaluated drags of the different samples and their relative positioning will be analyzed in relation to their surface features, and it will be demonstrated that all the pertinent roughness parameters are to be taken into account to explain data that could seem unexpected.

\section{Experimental test set-up, samples, and measurement techniques}

\section{A. Experimental test set-up and aerodynamic conditions}

The experimental investigations were conducted in a wind tunnel named S8Ch in the Fluid Mechanics Laboratory based in Meudon, France. It belongs to the Aerodynamics, Aeroelasticity, and Acoustics Department of ONERA. This facility is an open-circuit model which can generate subsonic, transonic and supersonic flows in continuous conditions. Stagnation conditions $\left(P_{i 0}, T_{i 0}\right)$ are close to the atmospheric pressure and room temperature ( $\left.293 \leq T_{i 0}(\mathrm{~K}) \leq 305\right)$. The flow in the tunnel is provided by a group of suction pumps with discharge in the atmosphere. The test section is $120 \mathrm{~mm}$ large, $100 \mathrm{~mm}$ high for a length of $500 \mathrm{~mm}$. The experimental set-up is shown in Fig. 2 and Fig. 3.

The bottom wall, starting at $X=0$, consists of three elements: a smooth wall $250 \mathrm{~mm}$ long, followed by the sample support wall which is $280 \mathrm{~mm}$ long, and finally the wall connecting the test section to the divergent. It should be noted that the connections between the three elements are adjusted in order to avoid parasitic steps. The support flat plate exhibits a $200 * 60 \mathrm{~mm}^{2}$ hole in which the rough surface samples are then inserted. As a consequence, and due to these dimensions, the boundary layers of the wind tunnel lateral walls are not supposed to interfere with the measurements focused only on the sample. As it can be observed, the smallest flat plate - i.e. the sample - is directly connected to a three-component balance, and a tiny gap $(0.4 \mathrm{~mm})$ exists between the sample and its surrounding support plate to ensure no contact disturbance during measurements. Moreover, the sample and the plate around it are mounted as flush as possible. The device used to fix the sample on the Kistler balance is equipped with altitude settings (2 upstream, 1 downstream) to ensure the continuity of the sample with its support. Besides, to maintain the equality between the pressures of the lower cavity (pressure $\approx \mathrm{Pa}$ ) and the test section, and therefore to avoid unwanted vertical forces, a suction pump is used (the hole is visible on the right side of the lower cavity). 


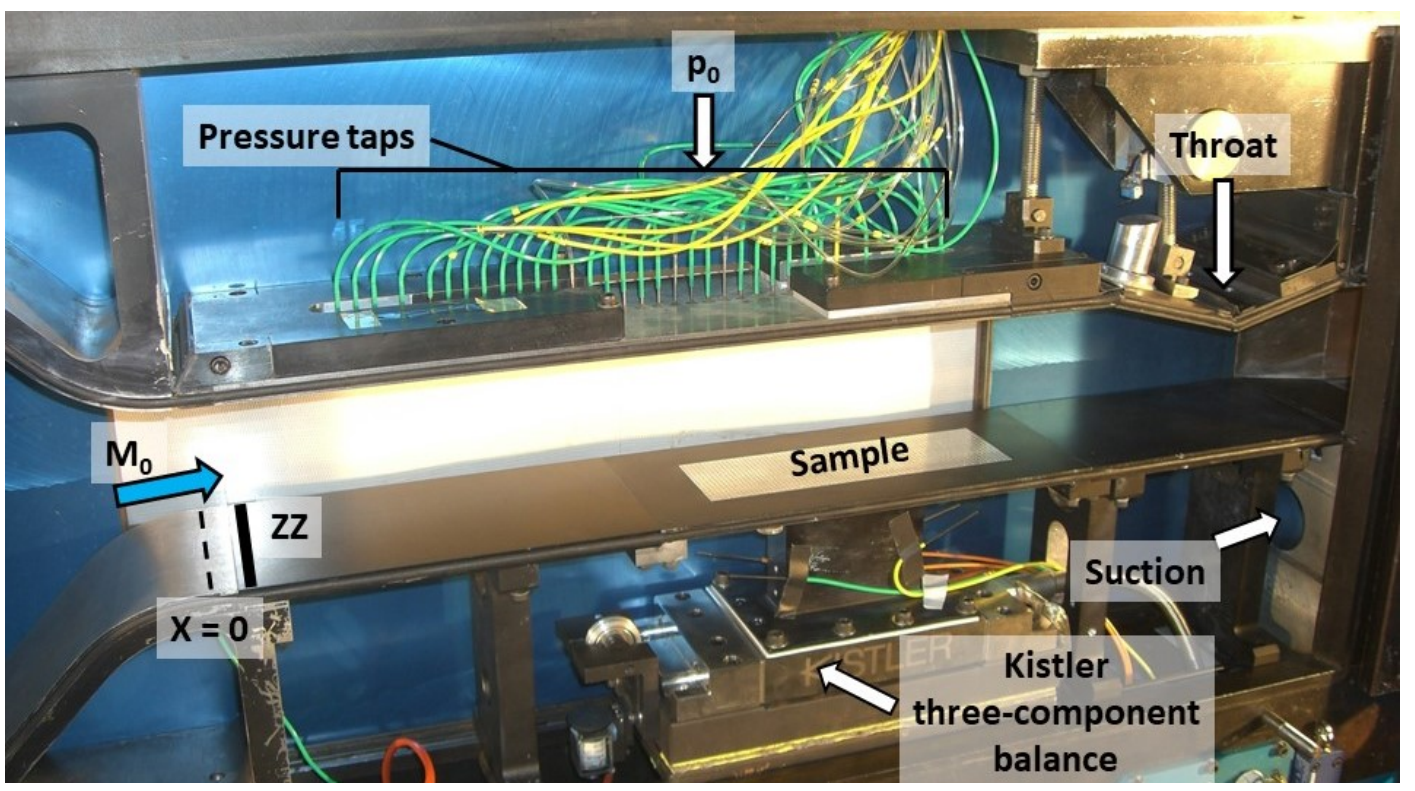

Fig. 2 Experimental set-up in the S8Ch wind tunnel.

The upper wall is equipped with 33 pressure taps $(0.4 \mathrm{~mm}$ diameter, linked to multi-sensors Pressure Systems EPS-32 HD of +/- 15 Psid), and the bottom wall with 10 taps located downstream of the sample (not visible in Fig. 2). Measurements obtained from these devices allow the evolution of the flow velocity in the test section to be controlled. The Mach number $M_{0}$ is tuned by the variation of the throat section and calculated from the pressure $P_{i 0}$ and the pressure tap $p_{0}$ located in front of the leading edge of the sample at $X=290 \mathrm{~mm}$. The boundary layer transition is triggered by means of a "ZZ" tape located at $X=20 \mathrm{~mm}$. This zig-zag turbulator is $6 \mathrm{~mm}$ wide, for a thickness of $0.1 \mathrm{~mm}$. 


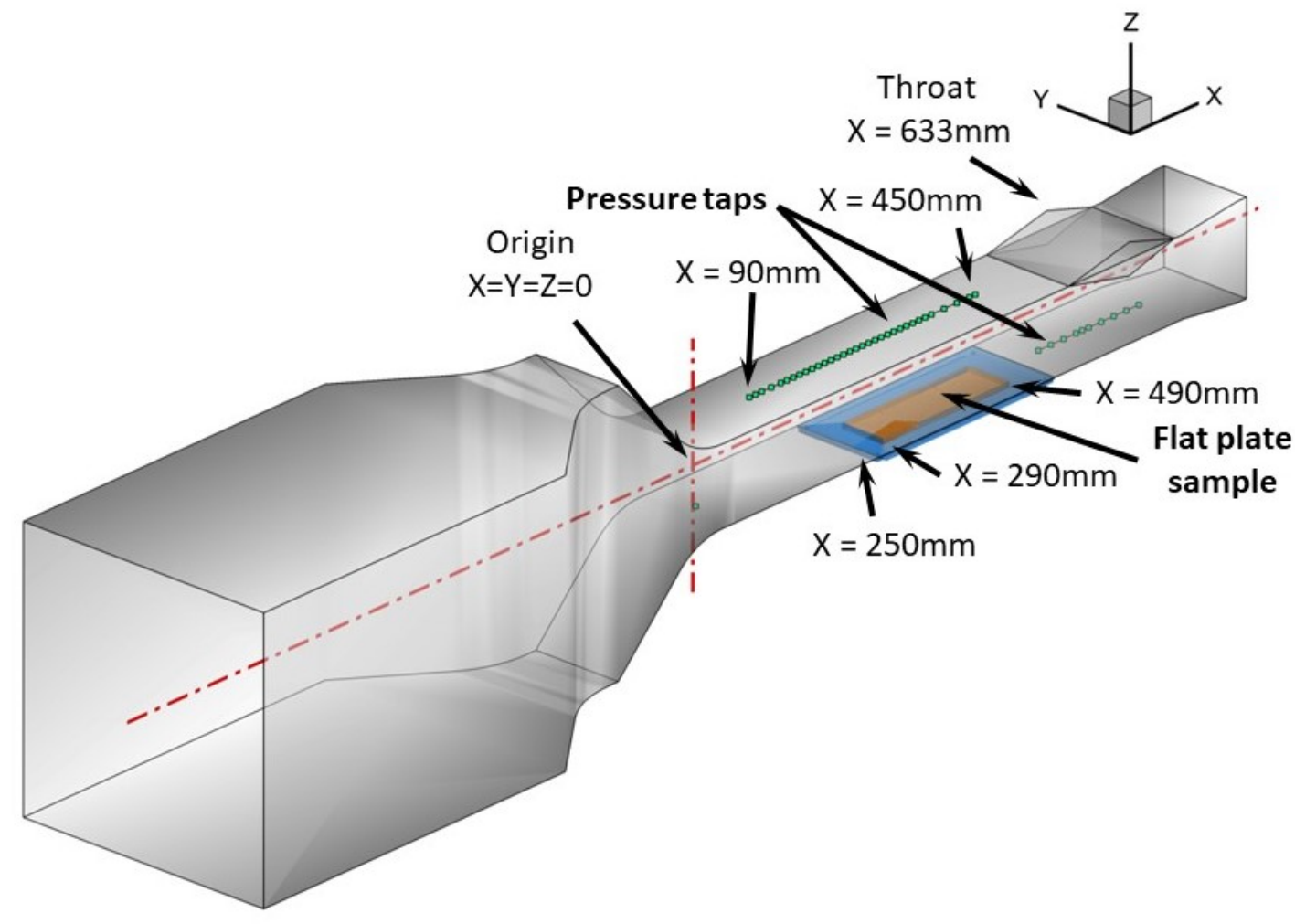

Fig. 3 Sketch and coordinates of the experimental setup.

The experiments were performed for Mach numbers extending from 0.55 to 0.8 and Reynolds numbers based on the sample length from 2.1 to 2.6 million. The test parameters $\left(P_{i 0}, T_{i 0}, M_{0}\right.$, static pressures on the upper wall, etc.) were acquired for each measuring point. Fig. 4 shows the evolution of the Mach number on the upper wall of the test section for the nominal Mach numbers $M_{0}$ retained in this study. The hatched vertical lines represent the position of the sample. These results show a constant distribution of the Mach number in the part of the test section where the sample is located. 


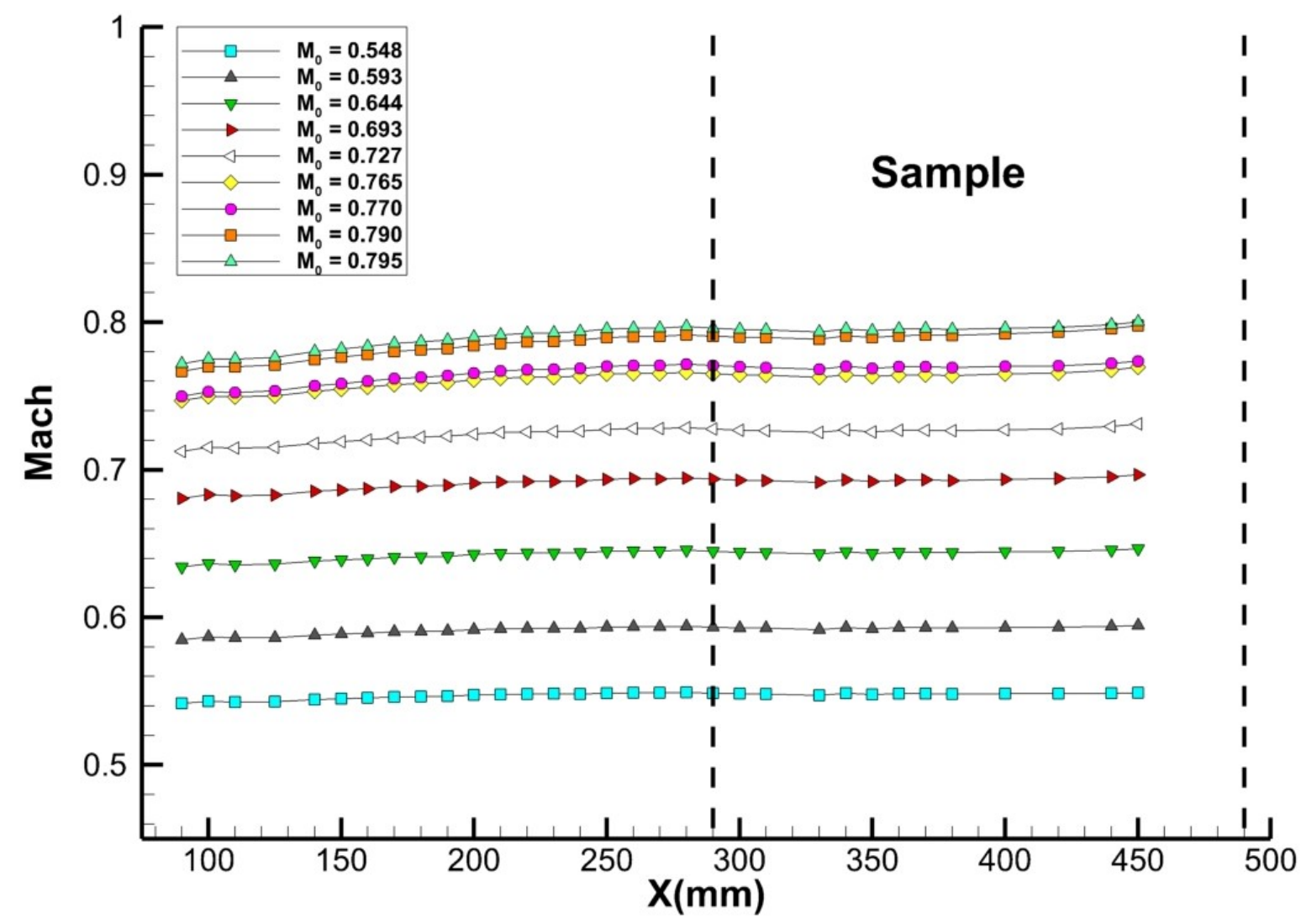

Fig. 4 Evolution of the Mach number on the upper wall of the test section.

\section{B. Samples of different roughness levels}

\section{Roughness parameters}

A real surface, whatever its manufacturing process, is not perfectly smooth. It is made of innumerable microscopic or macroscopic imperfections. These so-called imperfections are defined in comparison with a "virtual" average surface and can be therefore considered as peaks or as valleys. They constitute the roughness, which is in many fields a crucial parameter to characterize a given surface: drag production or reduction capabilities of course, but also resistance to wear, to corrosion, available contact or exchange properties...

The surface roughness can be quantified by a large number of parameters which are usually extracted from roughness profiles obtained via measurement tools such as optical or by-contact roughness meters. The latter are equipped of a moving stylus touching the considered surface to scan a straight line that constitutes the primary profile. A filter is applied to this profile to dissociate roughness from undulation and statistical formulas are then 
used to calculate the different surface characteristics. It is of note that among these roughness parameters, the most relevant are not the same in all the application fields. Table 1 gathers the ones generally used in the domain of external Fluid Dynamics and more specifically in this study.

The parameter of average roughness $R a$ is probably the most common. It represents the arithmetic average value of the filtered roughness profile determined from deviations about the "virtual" center line within the evaluation length. It gives an overall but yet useful information about the general roughness level of the surface. $R q$, or $R r m s$, is the root mean squared parameter, it is comparable to $R a$. Another interesting parameter is the average profile height $R z$ which corresponds to the mean of peak to valley amplitudes $(R p+|R v|$, if $R p$ and $R v$ are respectively the maximum peak height and valley depth) over five sampling lengths. $R z$ can be five to ten times greater than $R a$ for a given sample. Then, $S k$ and $K u$ are two additional statistical parameters that can be considered. $S k$ is the skewness coefficient giving the asymmetry of the amplitude distribution curve: if $S k$ is positive, the surface shows more peaks or very high peaks compared to the valleys and vice versa. While $K u$ is the kurtosis coefficient that provides information about the shape of the amplitude distribution curve: it has a value of 3 for a Gaussian distribution, below 3 if the amplitude distribution curve is narrower than a Gaussian (more homogeneous), and above 3 in the case of samples showing peaks or valleys with relatively great or intermediate amplitudes more numerous than they would be in a Gaussian distribution. Finally, $S p$ is the average absolute slope parameter, it is related to the shape of the roughness elements, and so to the way the flow "sees" these elements. It is the discrete form of the effective slope mentioned in the introduction. The sum for the calculation of $S p$ is done on all the points of the complete sampling length (see the next paragraph).

Table 1 Main roughness parameters and their definitions

\begin{tabular}{|c|c|c|}
\hline$R a=\frac{1}{l_{r}} \int_{0}^{l_{r}}|Z(x)| d x$ & $R q=\sqrt{\frac{1}{l_{r}} \int_{0}^{l_{r}} Z^{2}(x) d x}$ & $R z=\frac{1}{5} \sum_{1}^{5}(R p(i)+|R v(i)|)$ \\
\hline$S k=\frac{1}{R q^{3}}\left[\frac{1}{l_{r}} \int_{0}^{l_{r}} Z^{3}(x) d x\right]$ & $K u=\frac{1}{R q^{4}}\left[\frac{1}{l_{r}} \int_{0}^{l_{r}} Z^{4}(x) d x\right]$ & $S p=\frac{1}{N} \sum_{1}^{N-1}\left|\frac{y(i+1)-y(i)}{x(i+1)-x(i)}\right|$ \\
\hline
\end{tabular}




\section{Characteristics of the samples}

In this article, eight samples made of aluminum alloy are presented. Contrary to some academic surfaces, these eight samples ( $k$-type) all show dense distributions of rough elements. They can be arranged into four groups:

- Group 1: Bare metal samples corresponding to machining methods (RS, MP),

- $\quad$ Group 2: Paint samples (PS1, PS2),

- Group 3: Pressure sensitive paint samples (PSP1, PSP2),

- $\quad$ Group 4: Abrasive paper samples (P1000, P280).

The determination of roughness parameters was made using a feeler device of the Mitutoyo brand. The evaluation length is set at $12.5 \mathrm{~mm}$ (8,333 measuring points, i.e. one point every $1.5 \mu \mathrm{m})$, the movement speed is 0.5 $\mathrm{mm} / \mathrm{s}$, and the "cut-off" is equal to $2.5 \mathrm{~mm}$. Several line scans were performed on each sample (at least two in the longitudinal direction, and one along the transversal axis) to assess the surface homogeneity. Each overall profile is then divided into five sampling lengths, as indicated above. The results are presented in Table 2. It summarizes the different relevant roughness parameters of this set of samples.

The reference sample RS, in first position, corresponds to a standard machining finish (for instance for noncryogenic wind tunnel models) with an average roughness height $R a$ rather low of $0.68 \mu \mathrm{m}$, an average profile height $R z$ of $5.6 \mu \mathrm{m}$, and a very low slope value of 0.051 . Its roughness profile in Fig. 5A shows peaks globally larger than valleys but also numerous valleys and some of them very deep. As a consequence, its $S k$ coefficient is slightly negative and $K u$ is a bit above 3 .

The machining of the sample MP is identical to the previous sample RS, and then the plate is sanded with increasingly fine papers to achieve a mirror polish finish. The average roughness height that is achieved is $0.23 \mu \mathrm{m}$, as well as an extremely low $R z$ of $1.5 \mu \mathrm{m}$, the slope value being half the one of RS: 0.025 . The roughness profile in Fig. 5B shows equally distributed peaks and valleys of less than one micrometer. It leads to a very narrow amplitude distribution curve, a $S k$ value really close to 0 , and a $K u$ coefficient below 3 .

PS1 and PS2 samples are covered with a mat white paint, and then sanded with a P1500 paper for PS1 and a P800 for PS2. PS1 has a very low average roughness height of $0.26 \mu \mathrm{m}$ and a slope value equivalent to the one of RS. PS2 shows a $R a$ parameter close to $0.4 \mu \mathrm{m}$ and its slope is higher: 0.081. Fig. 6 gives the roughness profiles of PS1 and PS2. It can be observed that the sanding action has eliminated the greatest peaks but not the valleys which 
are more numerous and also greater. That explains the negative values of $S k$ for both samples as well as $\mathrm{Ku}$ coefficients clearly stronger than 3.

Table 2 Sample roughness characteristics

\begin{tabular}{|c|c|c|c|c|c|c|c|c|}
\hline & $\mathbf{R a}(\boldsymbol{\mu m})$ & $\mathbf{R z}(\boldsymbol{\mu m})$ & $\mathbf{R q}(\boldsymbol{\mu m})$ & $\mathbf{R p}(\boldsymbol{\mu m})$ & $\mathbf{R v}(\boldsymbol{\mu m})$ & $\mathbf{S k}$ & $\mathbf{K u}$ & $\mathbf{S p}$ \\
\hline $\mathbf{R S}$ & 0.68 & 5.6 & 0.82 & 1.9 & 3.7 & -0.26 & 3.66 & 0.051 \\
\hline MP & 0.23 & 1.5 & 0.28 & 0.73 & 0.8 & -0.06 & 2.52 & 0.025 \\
\hline PS1 & 0.26 & 2.43 & 0.34 & 0.90 & 1.5 & -0.62 & 4.14 & 0.058 \\
\hline PS2 & 0.40 & 3.4 & 0.52 & 1.25 & 2.1 & -0.64 & 3.77 & 0.081 \\
\hline PSP1-1 & 0.55 & 4.23 & 0.70 & 2.75 & 1.5 & 0.73 & 3.85 & 0.043 \\
\hline PSP1-2 & 1.1 & 10.5 & 1.55 & 7.5 & 3.0 & 1.54 & 8.48 & 0.056 \\
\hline PSP2 & 2.3 & 22.0 & 3.3 & 16.9 & 5.15 & 2.03 & 9.39 & 0.131 \\
\hline P1000 & 5.3 & 35.8 & 6.6 & 18.0 & 17.8 & 0.14 & 2.93 & 0.446 \\
\hline P280 & 11.8 & 66.9 & 14.5 & 33.6 & 33.3 & -0.05 & 2.46 & 0.47 \\
\hline
\end{tabular}

A pressure sensitive paint was applied on the samples PSP1 and PSP2. Then, they were covered with talc, which is a way of reducing the reflections on the model when images are acquired by cameras. For PSP1, Jet-fine talc $(<1$ $\mu \mathrm{m})$ from Imerys Talc recovers the PSP paint. The surface of this sample, and the one of PSP2 to some extent, is not as homogeneous as the surfaces of the previous samples: this is due to talc agglomeration. As a consequence, in Table 2, ranges are presented for PSP1, the roughness parameters depend on the line on which they are measured: $R a$ is between 0.55 and $1.1 \mu \mathrm{m}, R z$ between 4.23 and $10.5 \mu \mathrm{m}$, while the slope remains relatively limited with values from 0.043 to 0.056 . The two profiles of PSP1 exhibited in Fig. 7A are substantially different, the second one having an amplitude distribution curve much more extended and showing very high peaks (aggregated talc particles), the associated $S k$ and $K u$ values are particularly large in this case, 1.54 and 8.48 respectively. On the other hand, the PSP2 sample is covered with standard commercial talc (magnesium silicate hydrate). It has greater parameter values such as an average roughness height of $2.3 \mu \mathrm{m}$, a strong average profile height of $22 \mu \mathrm{m}$, and a slope of 0.131 . Here the talc particles are less fine and tend to gather as well and thus to form large structures as visible in Fig. 7B, with peaks higher than $20 \mu \mathrm{m}$. It implies levels of $S k$ and $K u$ coefficients that are the greatest of this set of samples: 2.03 and 9.39.

Finally, the samples P1000 and P280 are made of a metal plate covered with a commercial abrasive paper sheet. The P1000 paper is composed of silica grains of $18 \mu \mathrm{m}$ in diameter (1,000 grains per $\left.\mathrm{cm}^{2}\right)$, and the P280 paper is formed of $50 \mu \mathrm{m}$ grains $\left(280\right.$ per $\mathrm{cm}^{2}$ ). They are the roughest samples of this study: the parameters $R a, R z$, and $S p$ of 
P1000 are $5.3 \mu \mathrm{m}, 35.8 \mu \mathrm{m}$, and 0.446 respectively and the ones of P280 are $11.8 \mu \mathrm{m}, 66.9 \mu \mathrm{m}$, and 0.47. The profiles presented in Fig. 8 show for both samples a relatively good symmetry between peaks and valleys and also distributions really close to a Gaussian curve, leading to $S k$ values almost equal to 0 and $K u$ coefficients of about 3 .
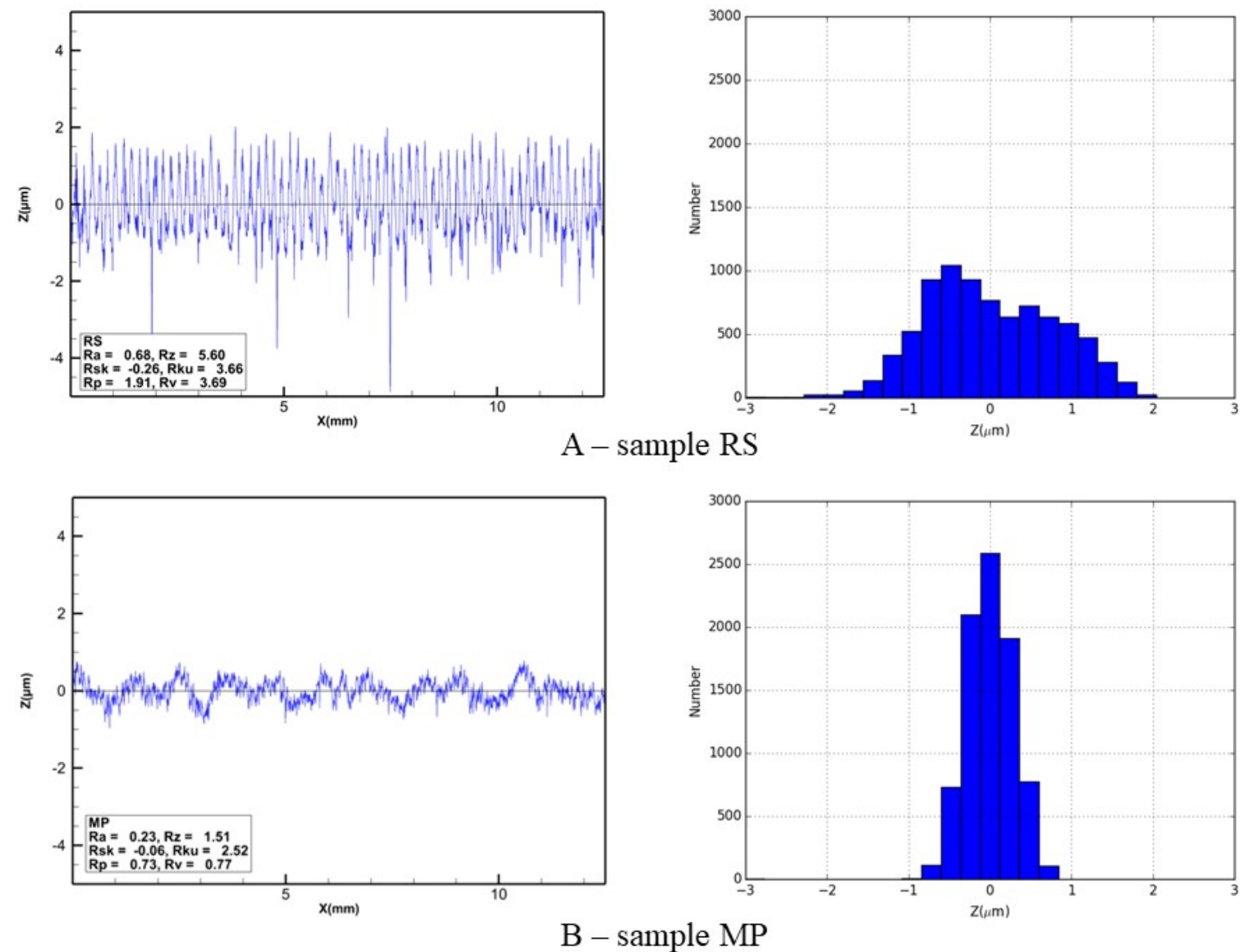

Fig. 5 Metallic samples: RS (A), MP (B), roughness profiles (left), histograms of amplitude distributions (right). 

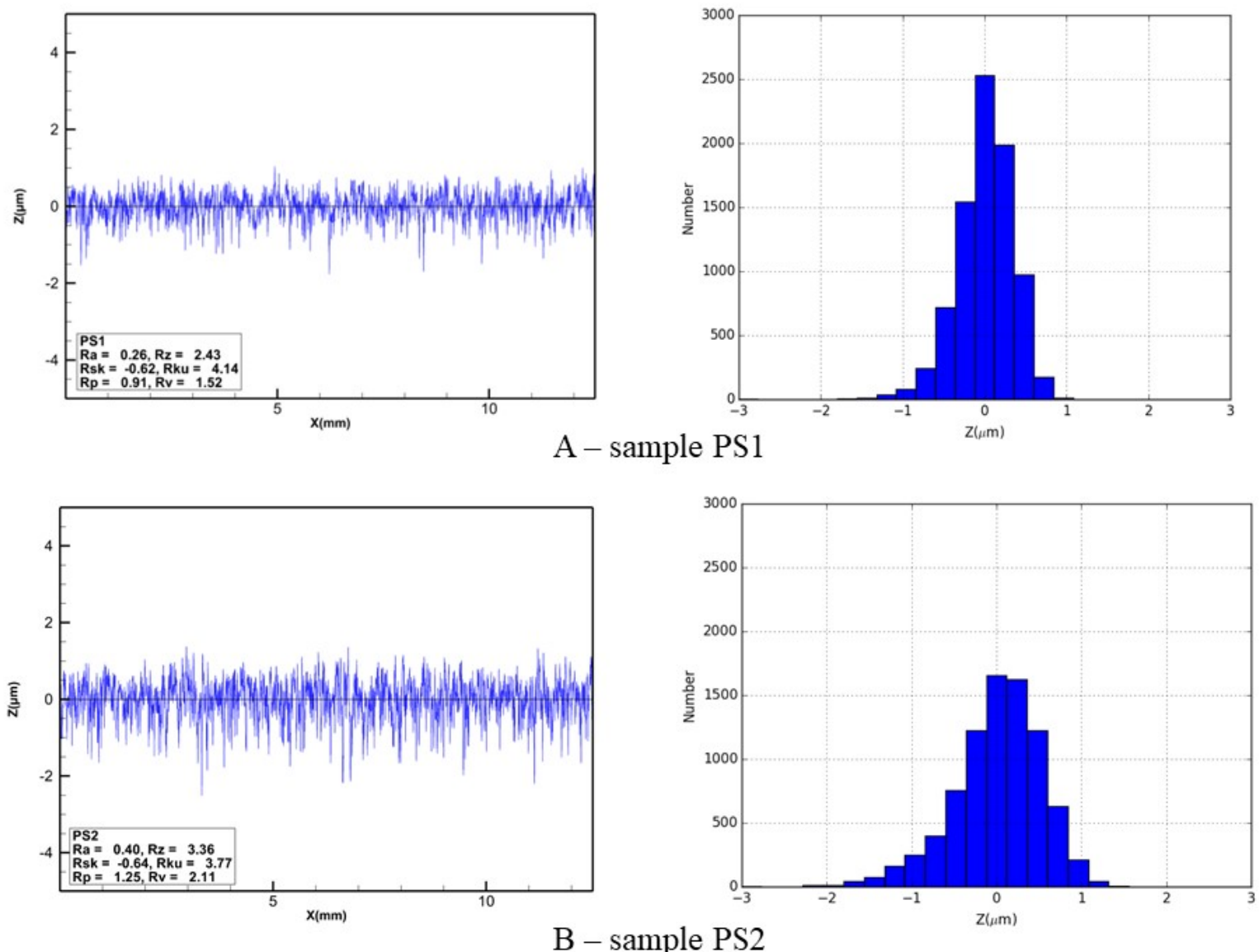

Fig. 6 Paint samples: PS1 (A), PS2 (B), roughness profiles (left), histograms of amplitude distributions (right). 

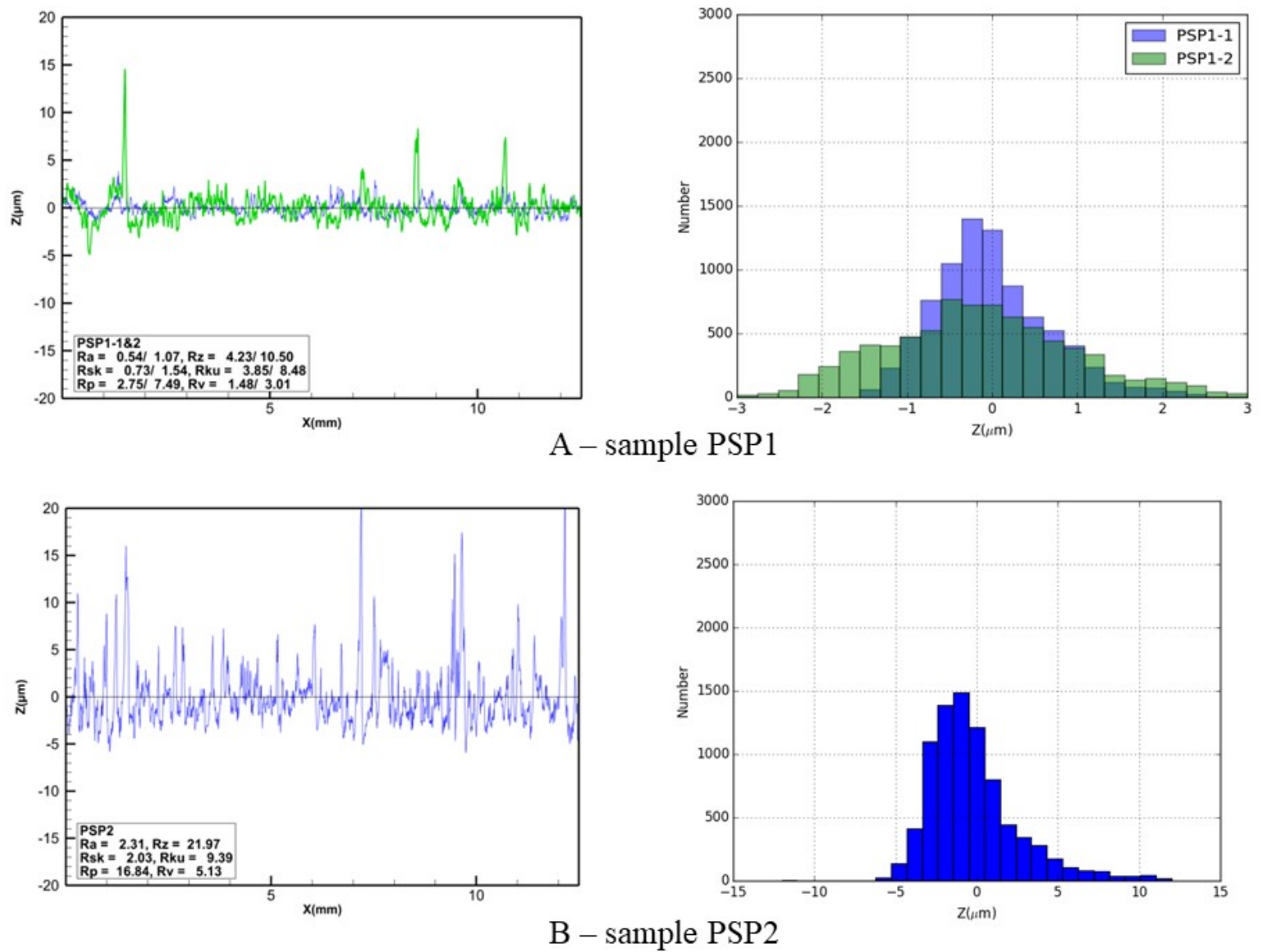

Fig. 7 PSP samples: PSP1 (A), PSP2 (B), roughness profiles (left), histograms of amplitude distributions (right). 

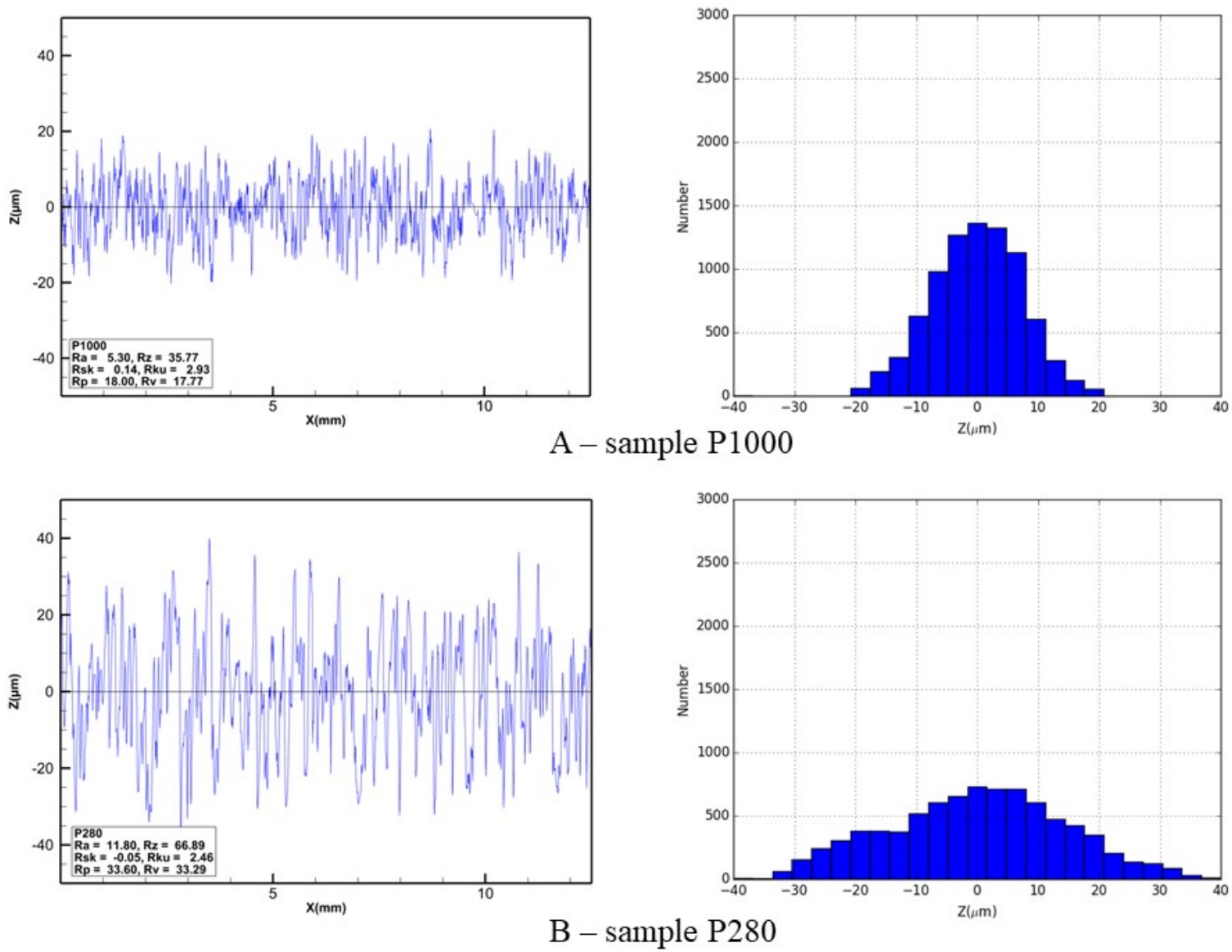

Fig. 8 Abrasive papers: P1000 (A), P280 (B), roughness profiles (left), histograms of amplitude distributions (right).

\section{Measurement techniques - Description and operational qualification}

In this study, two types of techniques were employed:

1) Measurement of stresses (micro-drag) with a three-component balance,

2) Measurement of velocity profiles with a two-component Laser Doppler Velocimetry system (LDV 2C).

\section{Measurement of stresses}

Evaluations of the drag forces were made using a dynamometric balance KISTLER type 9254 with three charge amplifiers type 5015A and a connection cable 1687B5. The system allows absolute measurements of the stresses along the three axes. It was calibrated before each use, and for the range $0-25 \mathrm{~N}$, the sensitivity is $-7.959 \mathrm{pC} / \mathrm{N}$, and the linearity is $0.03 \%$ for the full scale output. 
As a preliminary step, in order to control the balance, measurements of the longitudinal force (drag component) are made in the presence of known masses. During this test, successive masses of $50 \mathrm{~g}, 20 \mathrm{~g}, 10 \mathrm{~g}$ and $5 \mathrm{~g}$ are measured, and the signal is recorded. For example, Fig. 9 shows the time evolution of the longitudinal axis signal for a mass $M$ of five grams $(0.049 \mathrm{~N})$. At time $t \approx 25.6 \mathrm{~s}$, the mass is placed on the board and the delta which is obtained corresponds to a weight of $0.0489 \mathrm{~N}$, implying an accuracy of about $0.3 \%$ for this force intensity level which is representative of the sample drag differences that are to be observed.

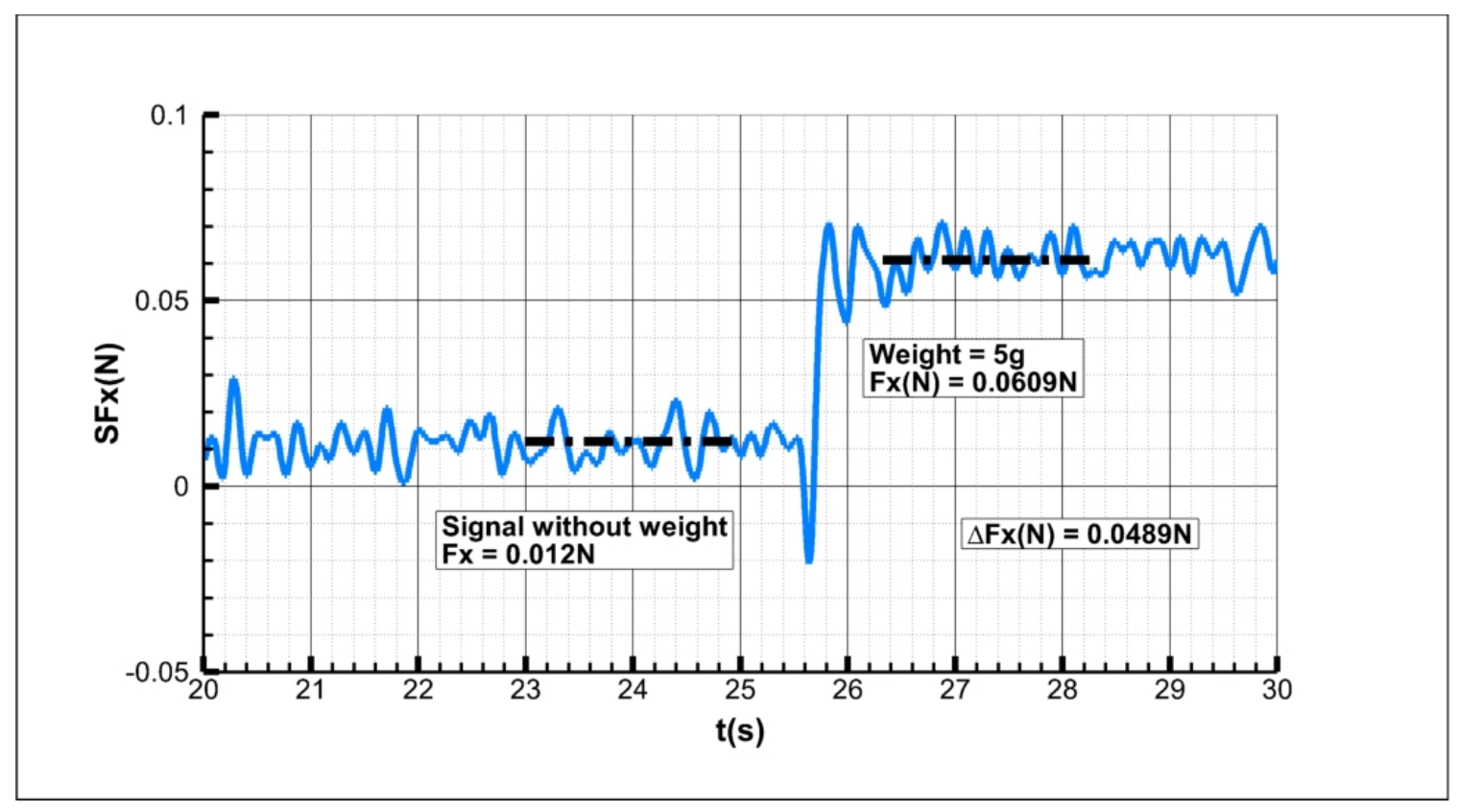

Fig. 9 Micro-drag forces qualification.

The measurement of the sample drags itself follows the protocol described below and presented in Fig. 10. In this figure, the black curve represents the evolution of the Mach number in the test section $\left(S M_{0}\right)$, the green curve is the longitudinal component $(S F x)$, the red curve is the transverse component (SFy), and finally the blue curve is the normal component $(\mathrm{SFz})$.

The different protocol phases for the acquisition of a measuring point are:

Phase 1 - Activation of the dynamometer amplifiers and recording of the initial conditions.

Phase 2 - Starting of the wind tunnel blower and suction pump. The flow in the test section is established, and the nominal Mach number is reached after about 35 seconds. Steadiness is achieved after about 40 seconds. 
Phase 3 - Pressures in the test section and in the lower cavity are equal, transversal and vertical parasitic forces are removed and the forces on the sample are constant and exploitable.

Phase 4 - After 70 seconds, the flow in the test section is stopped, and after a few seconds, initial conditions are again obtained. At 110 seconds, the recording is ended.

The quantification of the longitudinal stress is determined between states with (Phase 3) and without flow (Phase 1), and for each drag measurement point, the acquisition cycle was performed fifteen times. This procedure allowed the average of the force and the standard deviation to be calculated (see error bars in section IVA).

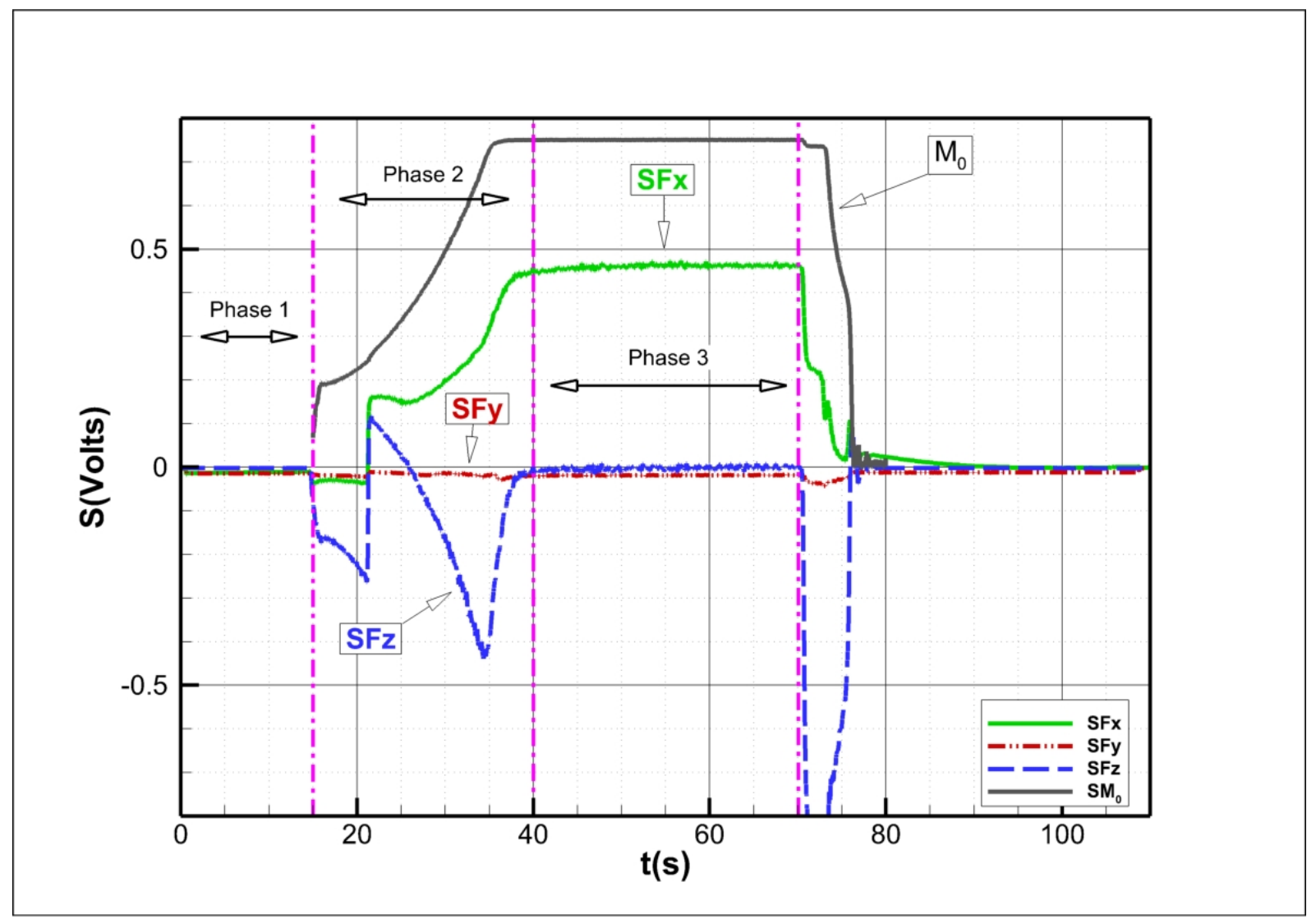

Fig. 10 Time evolution of the balance signals during drag acquisition.

\section{Measurements of velocity profiles}

To extend the investigation to local data, two-component LDV measurements were performed [18]. The system exhibits a specific mechanical configuration with a short focal which allows measurement points really close to the wall to be reached [19]. The optical setup is used in "forward scattering" mode. The emission side consists of a laser 
Spectra Physics 2,060 and a fiber beam splitter. The intersection of the two beam couples (blue and green) generates an $80 \mu \mathrm{m} \times 300 \mu \mathrm{m}$ measurement area (see Fig. 11). The latter is constituted of two networks of interference fringes. On the other hand, the optical receiver is based on Cassegrain telescopes collecting the light flux from each particle. The signal acquisition is carried out by means of a BSA F80, and raw recorded measurements are analyzed using the BSA Flow software. Besides, the flow is seeded with a generator located in the collector of the wind tunnel. The average size of the droplets (di-ethylhexyl sebacate) is about $1 \mu \mathrm{m}$. In this study, the mean and fluctuating velocity components were calculated by averaging over 20,000 particles for each measurement point (see Fig. 12). With this method, the velocity profiles were extracted at different positions on the sample in order to describe the flow conditions, and to characterize the boundary layer.

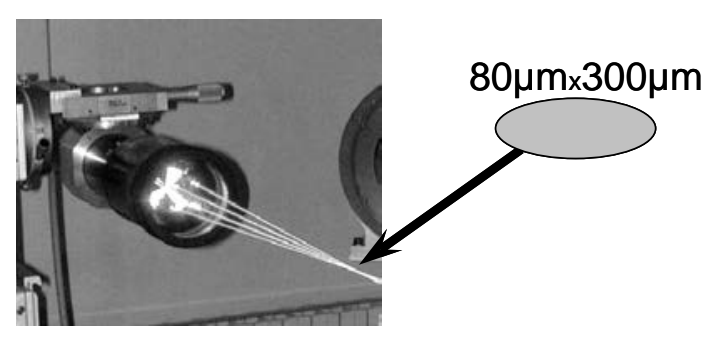

Fig. 11 LDV 2C: 4 laser beams constituting the measurement area.

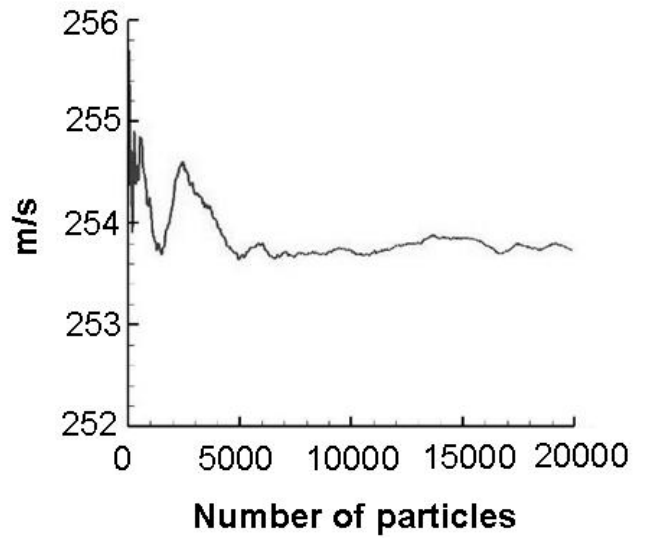

Fig. 12 LDV 2C: Statistical convergence of the velocity $\mathrm{U}(\mathrm{m} / \mathrm{s})$.

The objective of the LDV 2C measurements is to determine the parietal friction from measurements of boundary layer profiles and to derive information on the roughness of the wall. The study of the logarithmic region is applied for the treatment of the results [20]. The flow compressibility and the boundary layer thermodynamic characteristics are taken into account to extend the classical incompressible flow approach to transonic flows [21]. Laser Doppler Velocimetry measurements give access to the components of the velocity vectors and therefore to their mean values and fluctuations, but they do not give information on the thermodynamic quantity profiles such as temperature and density, so that iterative estimates are needed to determine the transformed speed.

On a smooth wall, the logarithmic region is governed, in compressible flow, by the relation: 


$$
\frac{u^{*}}{U_{\tau}}=\frac{1}{\kappa} \ln \left(\frac{y U_{\tau}}{v_{p}}\right)+C, U_{\tau}=u_{e} \sqrt{\frac{C_{f}}{2}}
$$

where $u^{*}$ is the longitudinal component of Van Driest's transformed velocity, $U_{\tau}$ is the friction velocity $\left(U_{\tau}=\left(\tau_{p} / \rho\right)^{1 / 2}\right.$ represents the tangential wall stress, $\rho$ the density), $v$ the kinematic viscosity, $y$ the distance to the wall. The index $p$ denotes the value at the wall and the index $e$ stands for external. The constants $\kappa$ (Von Karman constant) and $C$ have the following values: $\kappa \approx 0.41, C \approx 5.25$. For a boundary layer without pressure gradient, with $\delta$ its thickness, this logarithmic region ranges from $y^{+}=50$ to $y / \delta=0.1$.

On a rough wall, above the roughness sublayer, the logarithmic law is preserved but shifted $\left(\Delta u^{+}\right)[3]$ and the origin of the distances to the wall is unknown. Its expression can be extended to the form:

$$
\frac{u^{*}}{U_{\tau}}=\frac{1}{\kappa} \ln \left(\frac{\left(y-y_{0}\right) U_{\tau}}{v_{p}}\right)+C-\Delta u^{+}
$$

where $y_{0}$ represents the fictitious origin of the altitudes, generally located in the zone partially occupied by the rough elements, and $\Delta u^{+}$the downward shift of the logarithmic law, characteristic of the roughness effect.

Determination of the parietal friction coefficient is carried out by an iterative process. This method is implemented in an in-house code:

- An estimate of the offset (for example, half the average height of the rough elements) and of the friction coefficient is initially set. These values are used to determine the range of measurement points to be considered $\left(\left(y-y_{0}\right) U_{\tau} / v_{p}=50, y / \delta=0.1\right)$.

- For this set of measuring points, the offset is determined, which makes it possible to minimize the difference between the measurements and a linear law. This determination of the least squares line gives access to the other two unknowns, $U_{\tau}$ and $\Delta u^{+}$.

- This new determination of the offset and the friction velocity makes it possible to refine the range of measurement points to be considered. The process is resumed until this range of measurement points no longer changes. The friction coefficient is finally given by:

$$
\frac{C_{f}}{2}=\frac{\rho_{p} u_{\tau}^{2}}{\rho_{e} u_{e}^{2}}
$$


The LDV measurements were performed for the reference sample RS, and for the rough samples of silica grains P1000 and P280, at different $X$ positions and for Mach numbers equal to 0.6, 0.7, and 0.8.

\section{Numerical procedure: mesh generation, solver and methods}

\section{A. Structured grid}

To perform the RANS simulations which were aimed at being compared to the wind tunnel measurements described in the former section, a structured grid was generated with commercially available meshing software. An important aspect is that the surface roughness is not described by the grid: a unique grid is used for all the different samples. This is only a solver input which allows the roughness level to be modulated. Furthermore, the meshes are 2D grids. The computational volume covers the entire lower wall surrounding the considered sample but the whole wind tunnel is not calculated. Therefore, the CFD domain starts at $X=0 \mathrm{~mm}$ and the sample itself at $X=290 \mathrm{~mm}$. The total length and height (respectively $890 \mathrm{~mm}$ and 1,000 mm) were chosen large enough to avoid boundary issues. A global H-grid topology was adopted with three different blocks, the one in the middle corresponding to the sample (200 mm long). Point distributions ensure a grid refinement clearly satisfactory for RANS purposes. The boundary layer area is described by about 110 points in the normal direction with a first cell at one micrometer from the surface (leading to a posteriori checked normalized cell height $y^{+}$below 0.5 for the highest speeds) and a very limited vertical growth ratio inferior to 1.05 , as illustrated in Fig. 13. In the streamwise direction, the sample is discretized by about 100 points as well. Concerning the boundary conditions, the lower wall and flat plate are

defined as viscous walls, the upstream, upper, and downstream surfaces are defined as farfield conditions (as indicated, the wind tunnel effects are not taken into account). The global size of this grid is about 126,000 cells. 


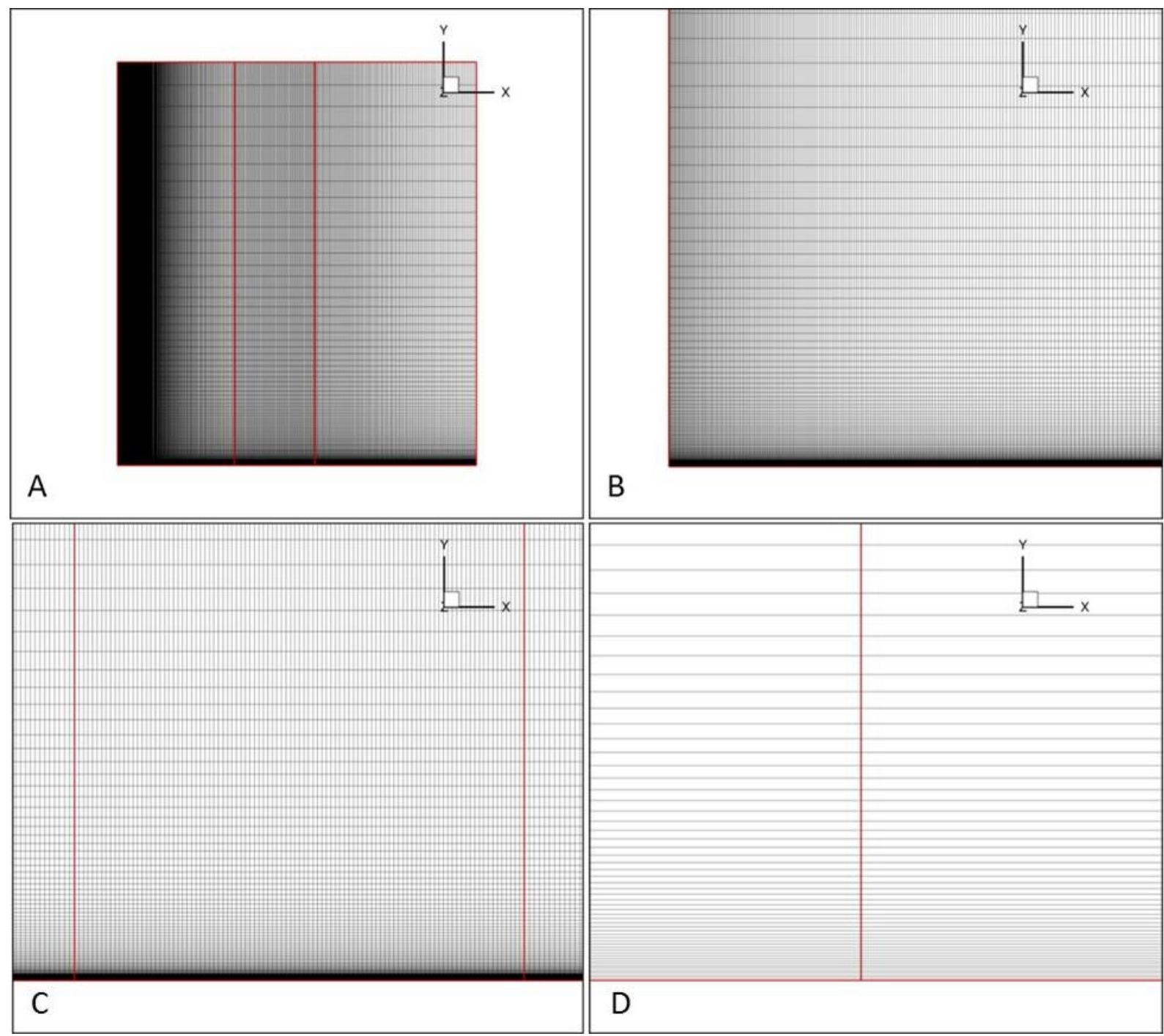

Fig. 13 Structured grid of the flat plate (A): zooms in the inflow (B), the sample (C), and the boundary layer resolution (D).

\section{B. ONERA-elsA solver}

The computations were carried out with the ONERA-elsA Navier-Stokes solver [22]. This software uses a cellcentered finite-volume discretization on structured, unstructured and hybrid meshes. Time integration is carried out by a backward-Euler scheme with implicit relaxation. Spatial discretization is realized using a second order accuracy central Jameson scheme with artificial viscosity [23]. Multigrid techniques are used to accelerate the convergence.

All the computations were performed in fully turbulent conditions. Turbulence effects are simulated by the twoequation Menter $k \omega$ shear-stress transport model [24]. Particular attention was given to the gradient computation and 
surface extrapolation (gradient computed at cell centers with a 5-point stencil and corrected to remove even-odd decoupling); these parameters are of prime importance for the evaluation of the friction drag. The 2D computations are realized in wind tunnel aerodynamic conditions $\left(M_{0}, P_{i}, T_{i}\right)$. In order to reach a satisfactory level of convergence, the computations were continued until the fluxes were stable enough to observe a drag variation inferior to one drag count over the last thousand iterations $\left(1 \mathrm{drag}\right.$ count $=10^{-4}$ ). More than 10,000 iterations were performed for each calculation.

\section{The equivalent sand grain roughness height method and the adaptation of an existing correlation}

Besides the general settings presented above, the method of the equivalent sand grain roughness height was used in the solver to model the roughness effects. This equivalent height corresponds to the virtual size of the uniformly packed sand grains tested by Nikuradse [3] that would produce the same frictional drag as the considered surface in the fully rough regime. Three models based on this approach are available in elsA. The one named AGC [25], for Aupoix-Grigson-Colebrook, was retained for this work. As explained, the unique input to be provided to the solver for a given surface sample is in this case the $k_{s}$ value. In 2004, Jiménez [1] rightly wrote that the notation $k_{s \infty}$ should be preferred, referring to the fact that this variable, which is then used to calculate the roughness Reynolds numbers

$k_{s}{ }^{+}$, is a property of the surface normally only valid in the fully rough regime. Keeping in mind this statement, the pragmatic idea of the numerical part of this study, mostly belonging to the transitionally rough regime, was to evaluate the available tools of the engineering field on this particular set of samples. And therefore, one of the necessary steps was to have a correlation relevant enough to reproduce the drag positioning of the different samples measured in the wind tunnel, or at least to know if a single correlation could give drag deltas between samples that would be consistent with the experimental ones. To avoid confusion, the notation $k_{\text {strans }}$ is adopted when it is necessary in the article.

Many correlations were tested, including the ones of Flack and Schultz based on $R q$ and $S k$ and the ones of Forooghi partially based on the slope parameter, as mentioned in the introduction, but none of them allowed the positioning of sample drags to be satisfactorily reproduced. This can probably be explained by the fact that most of the roughness levels considered in this work show really limited slope values, and as demonstrated by Flack and Schultz in [14], such low slope parameters - they indicate a threshold value of 0.35 - are associated with a so-called "waviness regime" in which the roughness function is strongly dependent on the slope and not anymore on the roughness height itself. Being aware of this and by considering that taking only the slope into account would be on 
the other hand insufficient, it was decided to focus on a Musker-type correlation which involves, as said above, not only the roughness height $R q$ and the slope $S p$ but also the skewness and kurtosis parameters, $S k$ and $K u$. Unfortunately, the original correlation of Musker [11], $k_{s}=R q\left(1+a^{*} S p\right)\left(1+b^{*} S k^{*} K u\right)$, where $a$ and $b$ are coefficients equal to 0.5 and 0.2 , does not give enough weight to the slope component and so it was adapted to better match the observations from the experiments and especially the relations between the drag positioning and the surface characteristics of the different samples. For the roughness levels with slope values inferior to 0.35 , the following formula has been applied:

$$
k_{\text {sTrans }}=\alpha * R q^{1 / 2 *}(1+100 * S p) *(1+0.2 * \operatorname{abs}(S k) * K u)
$$

where $R q$ in micrometers $(\mu \mathrm{m})$ is modulated by the use of the square root, the coefficient $a$ is dramatically increased to 100 to give a substantial role to the slope, the coefficient $b$ is kept, and an absolute value is added to $S k$ since it seemed that, in the present tests, the samples exhibiting more valleys did not produce less drag than the ones with more peaks, which is likely due to the absence of flow separation downstream of these very small roughness elements. Finally, $\alpha$ is a constant equal to $1 \mu \mathrm{m}^{1 / 2}$ to ensure the dimension homogeneity and a $k_{s T r a n s}$ value in micrometers. The authors are conscious of the limitations of such an adapted correlation built on a limited number of samples and moreover in the delicate transitionally rough regime. Once again, the idea here was a first order approach to assess some existing CFD techniques. The values of equivalent roughness heights given by this correlation were compared to the ones obtained with known correlations, such as the one of Schaffler, $k_{s}=8.9 R a$. For example, the metallic reference sample RS, whose $R a$ is $0.68 \mu \mathrm{m}$, has a $k_{s}$ value close to $6 \mu \mathrm{m}$ with the former correlation, and the one adapted from Musker leads to $6.6 \mu \mathrm{m}$. Of course, the Schaffler relation, which only takes the average roughness into account, cannot be in agreement for all samples with the Musker-type one that involves more advanced features. Nevertheless, a correlation based on the one of Schaffler was used in this study for the samples whose slope parameters are higher than 0.35, i.e. the commercial sandpapers. For the latter, the relation $k_{\text {sTrans }}=$ $12.5 R a$ has allowed the experimental results to be reproduced, this value of 12.5 being greater than 8.9 probably partly because in these experiments when the flow passes from the relatively smooth metallic lower wall of the wind tunnel to a sandpaper sample, the drag measured is slightly overestimated compared to a case in which all the lower wall would have the same roughness level as the sample. This situation is no longer true with the metallic and painted samples whose roughness characteristics are much closer to the ones of the lower wall. All the $k_{\text {sTrans }}$ values 
considered in this work for the different samples are gathered in Table 3. They range from about 2 to 150 micrometers.

Table $3 \quad k_{\text {sTrans }}$ values for the different samples $(\mu \mathrm{m})$

\begin{tabular}{|c|c|c|c|c|c|c|c|c|}
\hline Correlation & MP & RS & PS1 & PS2 & PSP1 & PSP2 & P1000 & P280 \\
\hline Musker-type & 1.9 & 6.6 & 6.5 & 9.6 & 15.0 & 122.9 & & \\
\hline Schaffler-type & & & & & & & 66.2 & 147.5 \\
\hline
\end{tabular}

\section{Roughness regime, Reynolds number $k^{+}$, and $R a / \delta$}

Beyond the need of determining equivalent roughness heights for the numerical part of the study, these values shown in the previous section now allow the range of roughness Reynolds numbers $k^{+}$to be estimated. Similarly to the one defined in the introduction:

$$
k^{+}=\left(U_{\tau} / v\right) * k_{\text {sTrans }}
$$

where $U_{\tau}$ is the friction velocity, $v$ is the kinematic viscosity. Table 4 gives the roughness Reynolds numbers over a Mach range of $0.55-0.8$ for three representative samples: the mirror-polished, the reference, and the harshest sandpaper ( $U_{\tau}$ is here extracted from CFD computations). This represents a $k^{+}$variation, from a bit below 1 to about 100, clearly situating most of the study, as already mentioned, in the transitionally rough regime. While the mirrorpolished sample can be very likely considered as hydrodynamically smooth, the sandpaper P280 might be on the opposite close to the frontier of the fully rough regime for the highest Mach numbers. Eventually, it is of note that all the metallic and painted samples show such low slope parameters that the waviness regime [14], where the frictional forces can overwhelm the pressure drag by far as in the transitionally rough one, is also to be considered.

Table $4 k^{+}$values for the samples MP, RS, P280 from $M_{0}=0.55$ to 0.8

\begin{tabular}{|c|c|c|c|}
\hline Variable & MP & RS & P280 \\
\hline $\boldsymbol{U}_{\boldsymbol{\tau}}(\mathbf{m} / \mathbf{s})$ & $\sim 7$. to 9.6 & $\sim 7.3$ to 9.9 & $\sim 9.4$ to 13.1 \\
\hline $\boldsymbol{U}_{\boldsymbol{\tau}} / \boldsymbol{v}\left(\mathbf{m}^{-\mathbf{1}}\right)$ & $\begin{array}{c}\sim 380,000 \\
\text { to } 450,000\end{array}$ & $\begin{array}{c}\sim 400,000 \\
\text { to } 465,000\end{array}$ & $\begin{array}{c}\sim 520,000 \\
\text { to } 615,000\end{array}$ \\
\hline $\boldsymbol{k}_{\text {sTrans }}(\mathbf{m})$ & $1.9^{*} 10^{-6}$ & $6.6 * 10^{-6}$ & $147.5^{*} 10^{-6}$ \\
\hline $\boldsymbol{k}^{+}$ & $\sim 0.7$ to 0.9 & $\sim 2.6$ to 3.1 & $\sim 76$. to 91. \\
\hline
\end{tabular}


Another interesting variable to be highlighted is the ratio between the boundary layer thickness and a roughness height parameter such as $R a$ or $R z$. In the wind tunnel S8Ch, according to the Mach number and in function of the exact position on the sample, the boundary layer thickness $\delta$ can vary between about 9 to $15 \mathrm{~mm}$. In this paragraph aimed at giving orders of magnitude, an intermediate value of $10 \mathrm{~mm}$ will be used. Considering the reference sample RS, the ratios $R a / \delta$ and $R z / \delta$ are respectively close to $0.007 \%$ and $0.06 \%$ (see Table 5), which is so limited that no effect on drag could have been expected. And for a sample with a roughness level much more important such as P280, the ratios are $0.1 \%$ and $0.7 \%$, showing that a surface with peak to valley amplitudes representing not even $1 \%$ of the boundary layer thickness can already produce levels of drag much greater than a smooth element, as will be demonstrated in the next section.

Table $5 \quad R a / \delta$ and $R z / \delta$ orders of magnitude for the samples MP, RS, P280

\begin{tabular}{|c|c|c|c|}
\hline Variable & MP & RS & P280 \\
\hline $\boldsymbol{R a} / \boldsymbol{\delta} \mathbf{( \% )}$ & 0.002 & 0.007 & 0.1 \\
\hline $\boldsymbol{R z} / \boldsymbol{\delta} \mathbf{( \% )}$ & 0.015 & 0.06 & 0.7 \\
\hline
\end{tabular}

All these ranges allow the different samples and flow conditions of this study to be seen in comparison with the work already available in the literature. The specificity here is the very low levels of roughness and especially of slope parameters tested in the air at transonic speeds.

\section{Results - Drag forces}

This section will first present the drag values obtained for each sample over the investigated Mach number range and then it will propose an analysis of these force values in regard to the sample roughness characteristics that are presented in part I.

\section{A. Experimental and numerical outcomes}

As a reminder, the Mach numbers evolve in this study from 0.55 to 0.8 and the stagnation conditions $\left(P_{i 0}, T_{i 0}\right)$ are close to the atmospheric pressure and room temperature. For clarity reasons, it was chosen to present the force results split into two different figures, the first one focusing on the metallic and painted samples (MP, RS, PS1, PS2, and PSP1), the second one showing the PSP samples and abrasive papers (PSP1, PSP2, P1000, and P280). The 
reference sample RS, as well as PSP1, are visible on both Fig. 14 and Fig. 15, so that a connection can be made between the figures (different scales) without overloading them. Experimental and numerical results are presented together in order to be easily compared and commented. The error bars obtained through the experimental procedure defined above are given for the wind tunnel data and it can be noticed that the uncertainty is relatively limited considering the very low level of the forces to be measured (typically one Newton).

A first observation of these figures shows that the evolution of the force according to the Mach number is close to be linear for most of the samples. This non-parabolic shape is linked to the fact that the decreases of the air density (from 0.99 to $0.84 \mathrm{~kg} / \mathrm{m}^{3}$ ) and drag coefficient, along with the increments in the Mach and Reynolds numbers, compensate the action of the velocity square in the force expression.

Then, what must strike in Fig. 14 is the significant difference between the force produced by the mirror-polished sample MP and the one of the reference sample RS. While it has been shown that RS could have been a priori considered as hydrodynamically smooth, given its estimated $k^{+}$value, it appears here that the drag it generates is clearly greater than the one of the sample MP, especially at the highest Mach numbers. Indeed, for values of Mach from 0.7 to 0.8 , the RS drag force is stronger than the MP one of about 5 to $10 \%$, which is substantial. This highlights the tough characteristic of the question of knowing whether or not a surface will be smooth enough not to cost extra drag in the targeted aerodynamic conditions. In this particular case, such a difference between MP and RS drags was not expected and it emphasizes the potential impact of even really low roughness on turbulent drag at high speeds.

Focusing now on the MP sample itself, it can be said that the force measured at $M_{0}=0.55$ is the lowest one with a value close to $0.6 \mathrm{~N}$, while at $M_{0}=0.8$ it is between 0.9 and $0.95 \mathrm{~N}$. For this sample, the force evolution is not linear and the difference in drag level between this sample and the other ones seems to increase with the Mach number.

The reference metallic sample RS and the painted sample PS1 arrive next, they generate almost identical force values over the whole Mach range, in spite of quite different average roughness heights. Then, an intriguing result is the behavior of the couple PS2 / PSP1. The two samples have their measured force evolution curves that cross each other. At low speeds, PS2 produces less drag than PSP1, as expected if the average roughness heights are considered. But for the highest Mach numbers (above 0.7), it is PSP1which generates a lower drag force than PS2. These points will be further discussed below.

Concerning the CFD data, all the samples in Fig. 14 were treated by the adapted Musker-type correlation. The RS 
and PS1 samples give same numerical results since their $k_{\text {sTrans }}$ values are almost identical. Globally, the absolute levels of computed forces are slightly underestimated, especially at the lowest Mach numbers and for the PS2 sample. However, the drag deltas are relatively well captured, the difference between the mirror-polished and reference samples in particular is consistent in both approaches. The CFD seems to partially reproduce the specific shape of the MP force evolution, but as the equivalent roughness height is the only input, it cannot predict what is observed between PS2 and PSP1: as the equivalent height value of PS2 is inferior to the one of PSP1, its computed drag remains lower at all Mach numbers.

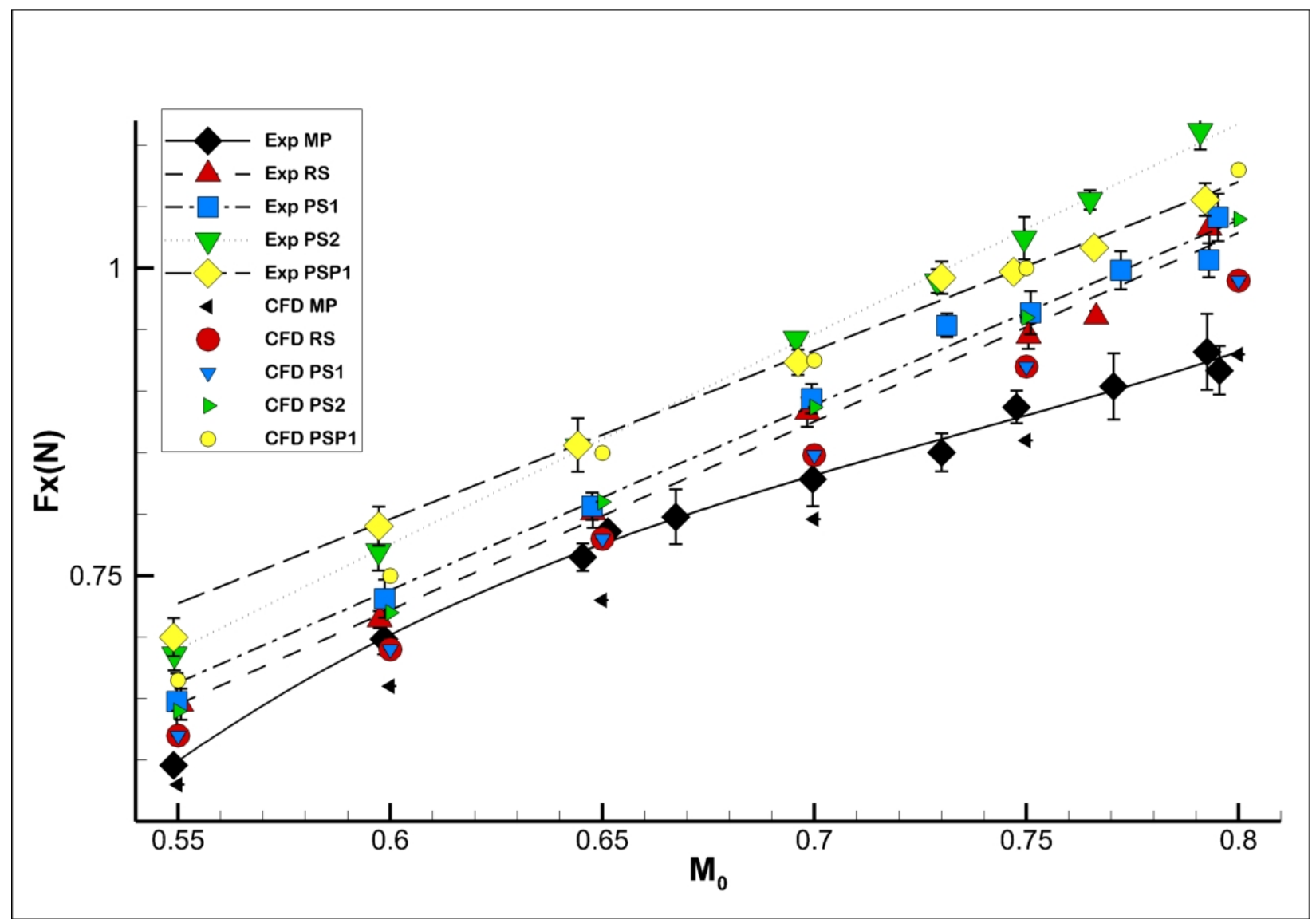

Fig. 14 Drag force evolution over Mach number range for the samples MP, RS, PS1, PS2, PSP1.

In Fig. 15, the PSP samples and abrasive papers are presented. The forces that are recorded are naturally much stronger and for the harshest sample P280, it ranges from about $1.0 \mathrm{~N}$ to $1.7 \mathrm{~N}$. What is surprising here is the position of the force curve obtained with the sample PSP2. It lies between the two sandpapers while its average roughness height is by far inferior to the ones of P1000 and of course P280. This unexpected outcome will be as well 
tackled in the next paragraph. The CFD computations, mixing in Fig. 15 results from Musker-type (RS, PSP1, PSP2) and Schaffler-type (P1000, P280) correlations, exhibit very good agreement with the experimental data for this sample group.

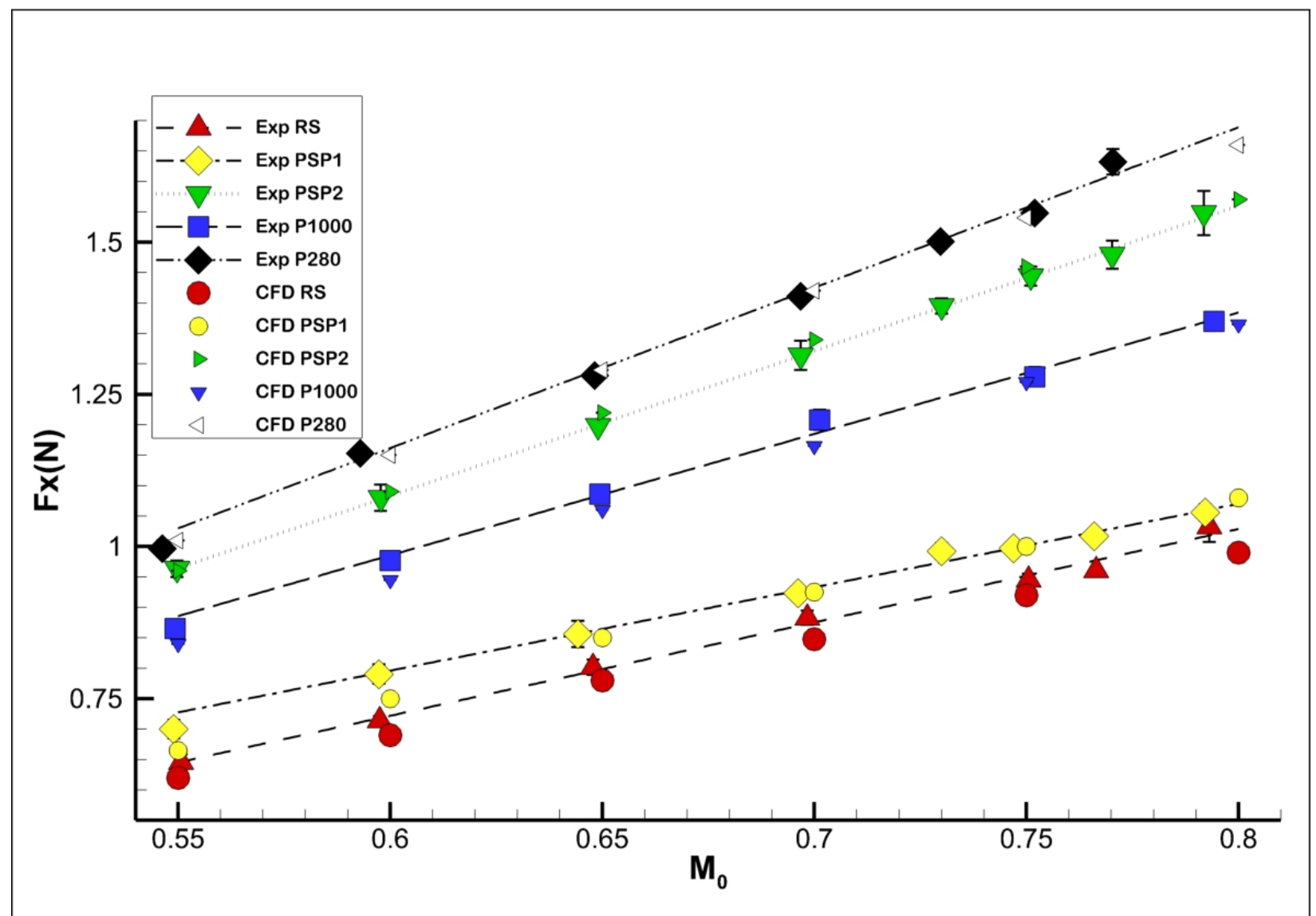

Fig. 15 Drag force evolution over Mach number range for the samples RS, PSP1, PSP2, P1000, P280.

As a conclusion on the use of the pragmatic numerical approach chosen in this study, it can be said the Muskertype correlation applied for slope parameters below 0.35 and coupled with the AGC model implemented in elsA has allowed drag deltas between samples globally consistent with the experimental ones to be obtained. And so, after complementary studies and potential improvements, it could be used in a more predictive way. Nevertheless, this method, still based on the equivalent sand grain roughness height, can fail in reproducing specific positioning situations in which the sample behaviors over the speed range are significantly different while their roughness characteristics lead to quite close $k_{\text {strans }}$ values (typically PS2 / PSP1). 


\section{B. Positioning of the different sample drags in relation with the roughness parameters}

Once the forces generated by all the different samples are known, it is then possible to order them by drag value and to analyze this positioning according to their roughness characteristics. As indicated above, the parameters that are considered as the most relevant in this study to predict or explain the drag penalty due to roughness are the following ones: an average height, here $R q$, but $R a$ could have been chosen as well, a slope parameter, here $S p$, and finally the skewness and kurtosis parameters $S k$ and $K u$. For reasons of consistency with the Musker-type correlation that was used, this latter couple of variables will be expressed here as a single one formulated as abs(Sk)*Ku . It is to be mentioned that this formulation, partially inspired by Musker, will not be suitable for samples that would have $S k$ values very close to zero, that is to say very symmetrical samples in terms of peaks and valleys, and strong $K u$ values, i.e. for example with an amplitude distribution curve much more extended than a Gaussian. Indeed, in this situation abs $(S k) * K u$ might be almost zero and so the influence of potentially high peaks and as deep valleys, even if taken into account by the other roughness parameters, could be underestimated by this approach. But in this work, that was not the case since all the samples showing $S k$ values close to zero have moderate $K u$ values. Otherwise, considering only $\mathrm{Ku}$ would have been an option. Actually, this type of surfaces exhibiting values of $S k$ really close to zero and very strong $\mathrm{Ku}$ parameters may not be so common in industrial applications. Anyway, in Table 6 the three variables $R q, S p$, and abs(Sk)*Ku are given for all samples in ascending order and juxtaposed with the drag positioning presented in the former section. As can be seen, the sample cells are colored by drag value.

And by observing the positioning of a given sample in each of the three groups $(R q, S p$, and $\operatorname{abs}(S k) * K u)$ in comparison with its positioning in term of measured drag level, some of the experimental outcomes that could seem surprising then become logical. For instance, the reference sample RS, second in term of drag value, does not show such a low $R q$ value (with $0.82 \mu \mathrm{m}$ it only gets the fourth position), but its slope value is the third one and its $\operatorname{abs}(S k)^{*} K u$ value is intermediate. As a consequence, it produces less drag than the samples PS1 and PS2 which have yet lower average roughness heights by far but whose slope and $\mathrm{abs}(S k)^{*} \mathrm{Ku}$ parameter values are significantly larger, mainly because in this case the polishing action on the paints did reduce or even eliminate the greatest peaks of their surface but not the valleys. Then, by considering the interesting sample PSP1 for which the $R q$ value is 1.12 $\mu \mathrm{m}$ (twice stronger than the one of PS2), it can be noticed that its drag is lower than the PS2 drag for Mach numbers superior to 0.66 and even close to the PS1 and RS drag level at Mach $=0.8$. This can be explained by the very low slope parameter of PSP1, second in the Sp positioning and only beaten by the mirror-polished sample, and in spite of 
a large value of $\operatorname{abs}(S k) * K u$. It underlines how the slope parameter can be predominant in such conditions, as already shown by Flack and Schultz [14]. Nevertheless, for greater levels of slope and abs(Sk)*Ku, it can be observed that this latter parameter is not to be forgotten because without taking it into account (or without taking into account at least $\mathrm{Ku}$ ), the drag positioning of a sample like PSP2 could not be understood. Indeed, PSP2 shows $R q$ and $S p$ values significantly lower than the ones exhibited by the sandpaper P1000 but its drag is however clearly higher. This is due to strong $S k$ and $K u$ values caused by the agglomerated structures of relatively big talc particles proper to the sample PSP2.

Table 6 Sample drag positioning according to the $R q, S p$, and abs(Sk)*Ku roughness parameters

\begin{tabular}{|c|c|c|}
\hline Ordered by $R q$ value & $R q$ & Ordered by measured drag value \\
\hline MP & 0.28 & MP \\
\hline PS1 & 0.34 & RS \\
\hline PS2 & 0.52 & PS1 (very close to RS) \\
\hline RS & 0.82 & PSP1(at high Mach number) \\
\hline PSP1 & 1.12 & PS2 (at high Mach number) \\
\hline PSP2 & 3.33 & P1000 \\
\hline P1000 & 6.59 & PSP2 \\
\hline $\mathrm{P} 280$ & 14.52 & $\mathrm{P} 280$ \\
\hline Ordered by $S p$ value & $S p$ & Ordered by measured drag value \\
\hline MP & 0.025 & MP \\
\hline PSP1 & 0.049 & $\mathrm{RS}$ \\
\hline RS & 0.051 & PS1 (very close to RS) \\
\hline PS1 & 0.058 & PSP1(at high Mach number) \\
\hline PS2 & 0.081 & PS2 (at high Mach number) \\
\hline PSP2 & 0.13 & $\mathrm{P} 1000$ \\
\hline $\mathrm{P} 1000$ & 0.45 & PSP2 \\
\hline $\mathrm{P} 280$ & 0.47 & $\mathrm{P} 280$ \\
\hline Ordered by abs(Sk)*Ku value & abs $(S k) * K u$ & Ordered by measured drag value \\
\hline $\mathrm{P} 280$ & 0.12 & MP \\
\hline MP & 0.15 & RS \\
\hline $\mathrm{P} 1000$ & 0.41 & PS1 (very close to RS) \\
\hline $\mathrm{RS}$ & 0.95 & PSP1(at high Mach number) \\
\hline PS2 & 2.41 & PS2 (at high Mach number) \\
\hline PS1 & 2.57 & P1000 \\
\hline PSP1 & 7.10 & PSP2 \\
\hline PSP2 & 19.1 & P280 \\
\hline
\end{tabular}


As a conclusion, without stating that all the rough sample behaviors in term of additional drag production can be anticipated or accurately predicted, it can be said that considering here the parameters $R q, S p, S k$, and $K u$ has allowed the qualitative drag positioning between samples to be fully explained.

\section{Results - LDV}

The analysis of the boundary layer profiles developing on the samples from the method described in section IC2 makes it possible to estimate the friction velocity $U_{\tau}$ and skin friction coefficient $C_{f}$ (Fig. 16). As a reminder, the sample is fixed on a three-component balance and surrounded by a $0.4 \mathrm{~mm}$ gap ensuring that there is no contact between the sample and the support. This gap, and for the abrasive papers the additional fact of switching from a relatively smooth wind tunnel wall to a rough sample, generate a disturbance in the boundary layer development. A certain distance is necessary for it to recover a usual flat plate form. Thus the rapid $C_{f}$ decreases of RS observed in Fig. 16, compared to the Ludwig-Tillman's law [21] for instance, are mainly due to this aspect of the experimental set-up. Nevertheless, the average $C_{f}$ values obtained by LDV for the different samples are in good agreement with the measured drag values of RS, P1000, and P280 shown in section IVA. The relative positioning and force level for each sample previously given by the balance are confirmed. For example, the average skin friction coefficient of RS in Fig. 16 can be estimated as close to 0.0029 at Mach $=0.7$, which leads to a force value of $0.85 \mathrm{~N}\left(F=0.5 \rho U_{e}^{2} S C_{f}\right)$ consistent with the data in Fig. 15. 


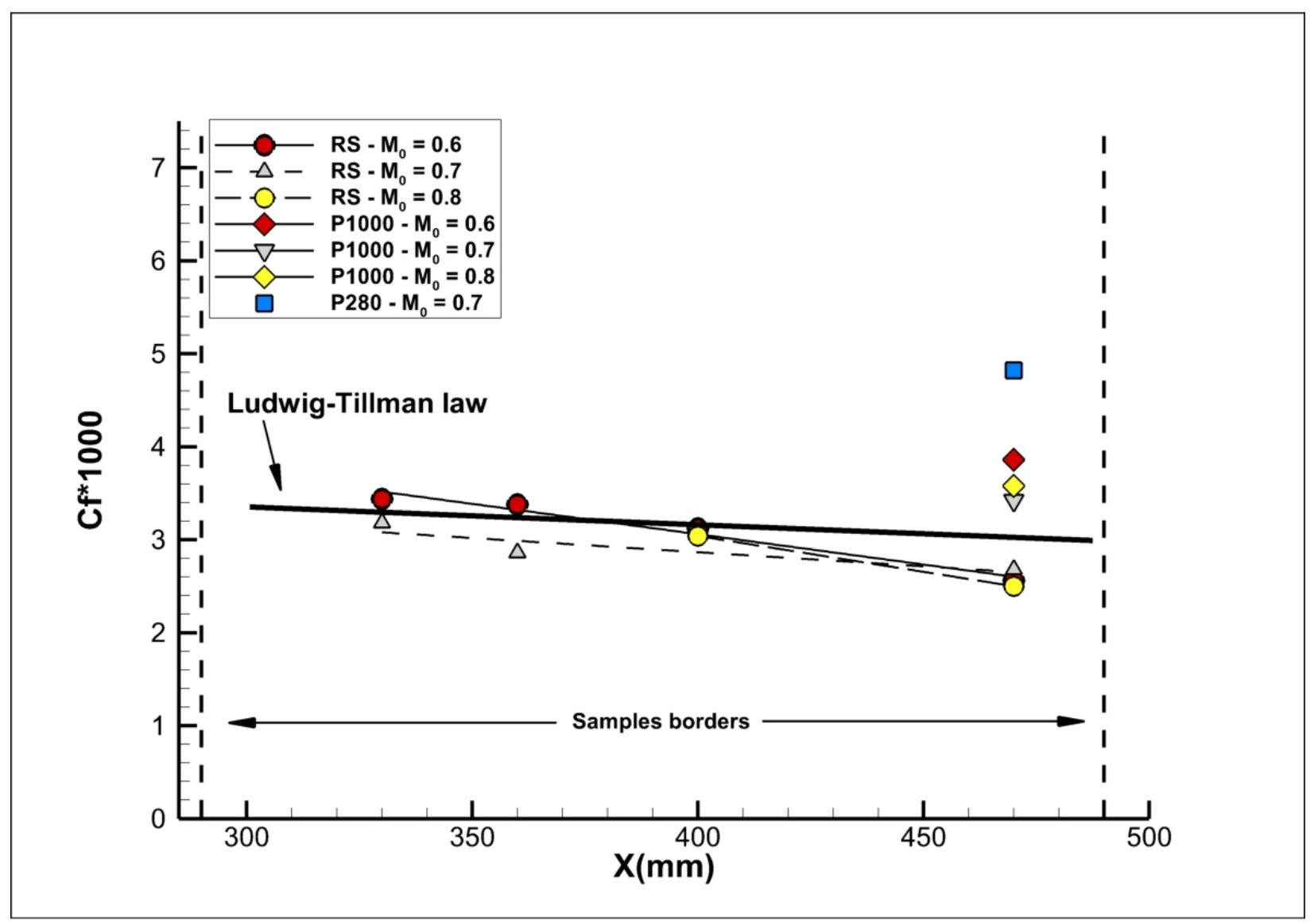

Fig. 16 LDV skin friction for RS, P1000, P280, $C f=f\left(X, M_{0}\right)$. 
The profiles of the longitudinal velocity component $U / U_{e}$ of RS, P1000, P280, on the downstream part of the sample at abscissa $X=470 \mathrm{~mm}$, for a Mach number $M_{0}=0.7$, are compared in Fig. 17 . The loss $\Delta u^{+}$mentioned in IC2 and affecting the boundary layer in presence of roughness is clearly tangible here. Furthermore, it can be noticed that the more the sample is rough the more the first accessible measurement point by LDV is far from the wall $\left(Z_{1}=\right.$ $0.114 \mathrm{~mm}, 0.143 \mathrm{~mm}, 0.22 \mathrm{~mm}$ for RS, P1000, and P280 respectively). At $Z=0.22 \mathrm{~mm}$, the sample P1000 shows a value $U / U_{e}$ decreased of about $13 \%$ compared to the reference sample, and the harshest P280 of more than $22 \%$. It demonstrates the massive impact of roughness on the logarithmic part of the boundary layer. However, roughness has little influence on boundary layer thickness and external flow and for these three sample profiles, the thickness $\delta$ is equal to about $13.5 \mathrm{~mm}$.

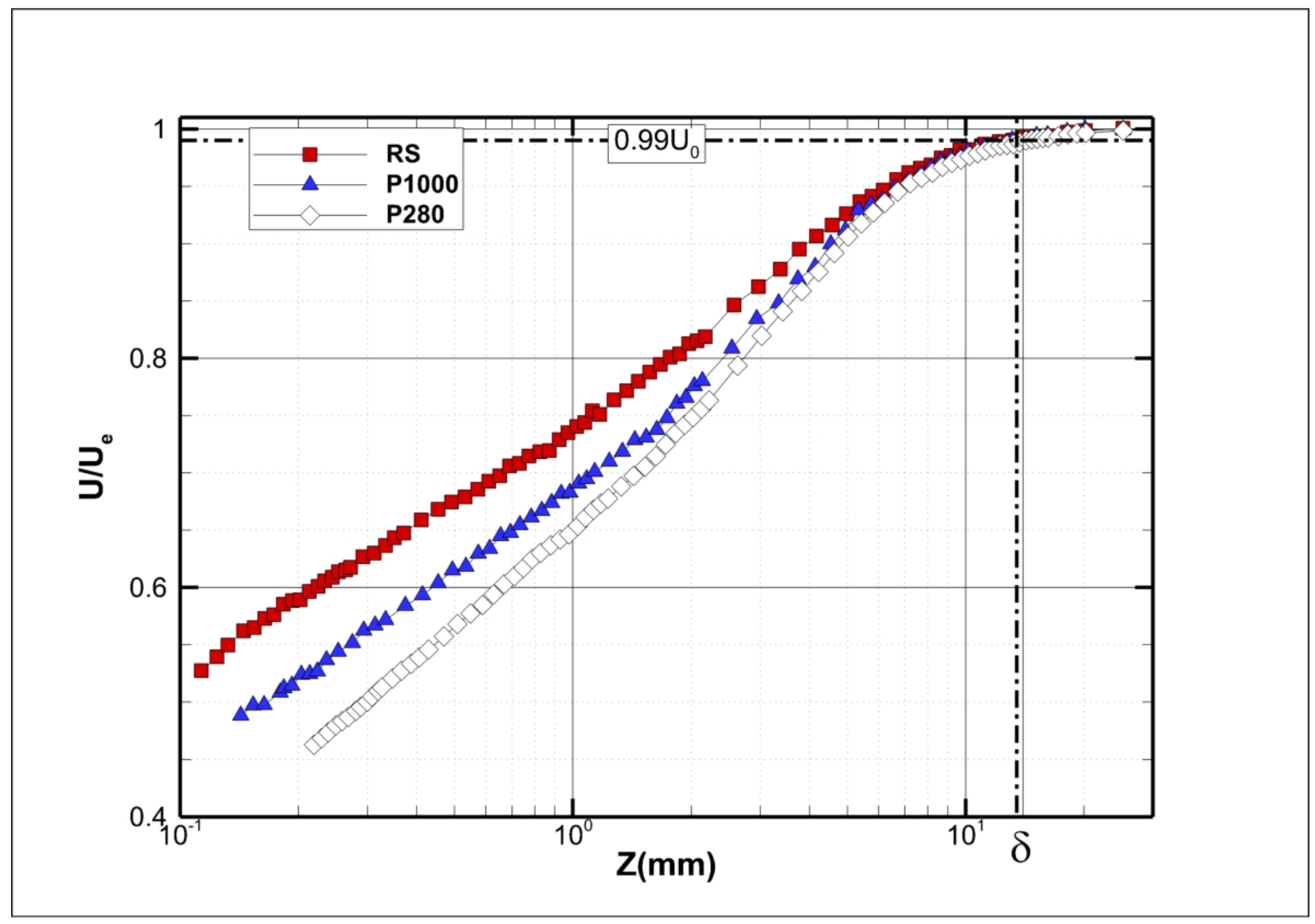

Fig. 17 Roughness effects on boundary layer profiles (LDV), $U / U_{e}=f(Z), M_{0}=0.7, X=470 \mathrm{~mm}$.

Then, in order to compare the $\Delta u^{+}$estimated from the balance measurements (via $F$ and $\sqrt{ }\left(2 / C_{f}\right)$ ) and from the LDV profiles, Fig. 18 gives the $U^{+}=\mathrm{f}\left(Z^{+}\right)$evolutions of the samples RS and P1000 at $M_{0}=0.7$ and $X=470 \mathrm{~mm}$ 
$\left(U^{+}=U / U_{\tau}, Z^{+}=\left(U_{\tau} / v\right) Z\right)$. It can be observed that the local $\Delta u^{+}$between these two samples at this abscissa is close to 3.87. Finally, the Table 7 presents the $\Delta u^{+}$obtained from the balance data between RS and P1000 (third column), these values show satisfactory agreement with Fig. 18 . And the $\Delta u^{+}-$strictly positive - of RS, calculated with MP as the smooth reference, is given as an additional illustration of the fact that the sample RS is not hydrodynamically smooth in these conditions.

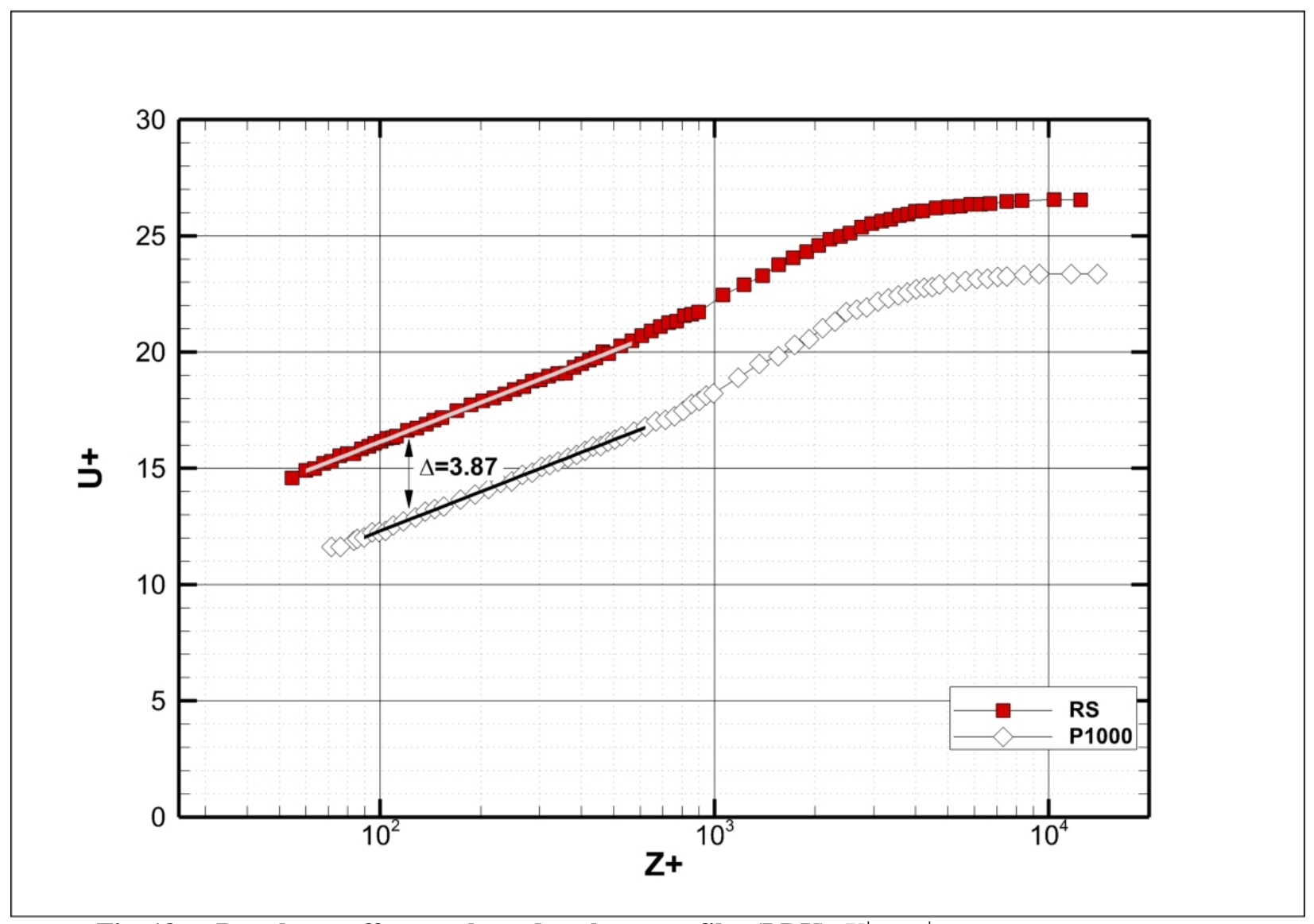

Fig. 18 Roughness effects on boundary layer profiles (LDV), $U^{+}=f\left(Z^{+}\right), M_{0}=0.7, X=470 \mathrm{~mm}$.

Table $7 \mathrm{Au}^{+}$from balance for samples RS and P1000 (cross-comparison with LDV)

\begin{tabular}{|c|c|c|}
\hline $\begin{array}{c}\text { Mach } \\
\text { number }\end{array}$ & $\mathrm{U}^{+}(\mathbf{M P})-\mathrm{U}^{+}(\mathbf{R S})$ & $\mathrm{U}^{+}(\mathbf{R S})-\mathrm{U}^{+}(\mathbf{P 1 0 0 0})$ \\
\hline 0.55 & 0.94 & 3.48 \\
\hline 0.60 & 0.27 & 3.63 \\
\hline 0.65 & 0.50 & 3.57 \\
\hline 0.70 & 0.77 & 3.70 \\
\hline 0.75 & 0.70 & 3.64 \\
\hline 0.80 & 1.27 & 3.39 \\
\hline
\end{tabular}




\section{Conclusions and prospects}

The present article was focused on the test data obtained from a campaign aimed at evaluating the effects of very low roughness levels on the flat plate turbulent drag at transonic Mach numbers from 0.55 to 0.8 . The experiments took place in the ONERA S8Ch wind tunnel: eight samples exhibiting different roughness characteristics, metals, paints and papers with $R a$ from 0.25 to $10 \mu \mathrm{m}$, were assessed in detail through measurement techniques including micro-drag quantification with a three-component balance and near-wall laser Doppler velocimetry.

Besides, this work mixed experimental and numerical approaches: a pragmatic CFD study, using a structured grid with the AGC model in the aerodynamic solver elsA, and based on the equivalent sand grain roughness height method and on a Musker-type $k_{s}$ correlation, was carried out to evaluate some of the available numerical tools.

These investigations were mainly situated in the transitionally rough regime, with roughness Reynolds numbers $k^{+}$ from below 1 to about 100. Also, most of the samples had very low slope parameters $S p$, typically much lower than 0.35 which is the threshold considered by Flack and Schultz, and therefore implying the waviness flow regime in which the friction forces overwhelm the pressure ones as well.

Finally, the outcomes of this study that should be highlighted are: first, the fact that the turbulent drag of a rough surface with roughness Reynolds numbers below the usual threshold values often considered in the engineering world (i.e. about 3.5 to 5) can be substantially larger than expected. In particular, the reference sample used here, whose $k^{+}$values of about 3 could have suggested that it would belong to the hydrodynamically smooth regime, actually produced drag forces 5 to $10 \%$ greater than the ones of the mirror-polished sample which exhibited $k^{+}$ below 1, for both experimental and CFD methods. Second, by a cross-analysis between surface drag production and roughness characteristics, it was demonstrated that taking into account only an average roughness height or only a slope parameter to predict the extra drag due to roughness was not enough: in this case, the four variables $R q$, $S p, S k$, and $\mathrm{Ku}$ had to be considered to explain a force positioning between samples a priori unexpected. And the predominance of the slope parameter in these regimes, already mentioned in the literature, has been clearly confirmed.

Another interesting aspect not mentioned in the body of the article and which might be the topic of a coming paper is the fact that most of the results from S8Ch, especially the ones involving the reference sample and PSP paints, are qualitatively in good agreement with results obtained with a much bigger sample - the ONERA Common Research 
Model [26] based on the NASA-CRM geometry used in the Drag prediction Workshop series [27] - in a much bigger wind tunnel: the ONERA S1MA (Modane), with its test section diameter of eight meters.

To conclude, this work can enlighten some aspects of the experimental versus numerical comparisons, especially for drag prediction purposes. Indeed, almost all the CFD computations, even the ones aimed at reproducing a test campaign, are carried out with perfectly smooth surfaces (except for the studies focused on icing issues or roughness effects on the laminar to turbulent transition). Yet, these results from S8Ch clearly showed that for samples or models which do not have a mirror-polished finish (and maybe still for them also, according to the quality of the finish and aerodynamic conditions), the drag produced at transonic Mach numbers can be significantly greater than it is for the infinitely smooth virtual surfaces used in calculations. And for a wind tunnel aircraft model, with a boundary layer thickness close to the leading edges of only several dozens of micrometers (much thinner than millimeters) and submitted to strong gradients, the roughness effects might be locally substantial and should be taken into account. As a consequence, even if of course other aspects such as transition triggering, geometry deformation, wind tunnel wall, and model support effects do play a major role, it is not impossible that some of the drag differences which have been observed in the past and until now between experimental and numerical sources come, in fact, from the roughness impact itself: a matter not enough considered, maybe somehow forgotten, and quite unknown still.

\section{Acknowledgments}

The study presented here was completed in the framework of ONERA general resources and partly in the research project "FROTTEMENT". The authors would also like to thank the ONERA team of the wind tunnel S8Ch, the DMPE team for its shared expertise, especially François Chedevergne, and the Modane team, in particular Aurélia Cartieri.

\section{References}

1.Jiménez, J., “Turbulent flows over rough walls,” Annual Review Fluid Mechanics, 36:173-96, 2004.

${ }^{2}$ Flack, K. A., Schultz, M. P., "Roughness effects on wall-bounded turbulent flows," Physics of Fluids 26, October 2014.

3.Nikuradse, J., “Laws of flow in rough pipes,” NACA Technical Memorandum 1292, 1933. 
${ }^{4 .}$ Colebrook, C. F., "Turbulent flow in pipes with particular reference to the transition between smooth and rough pipe laws,” J. Inst. Civ. Eng. 11, 133, 1939.

5.Moody, L. F., “Friction factors for pipe flow,” Trans. ASME 66, 671, 1944.

6.Flack, K. A., Schultz, M. P., Rose, W. B., “The onset of roughness effects in the transitionally rough regime,” International Journal of Heat and Fluid Flow, vol. 35, Pages 160-167, June 2012.

${ }^{7}$ Bradshaw, P., “A note on critical roughness height and transitional roughness,” Phys. Fluids 12, 1611-1614, 2000.

${ }^{8 .}$ Yuan, J., Piomelli, U., "Estimation and prediction of the roughness function on realistic surfaces," Journal of Turbulence, April 2014.

${ }^{9}$ Thakkar, M., Busse, A., Sandham, N. D., "Surface correlations of hydrodynamic drag for transitionally rough engineering surfaces,” Journal of Turbulence, 18:2, 138-169, 2017

${ }^{10}$ Thakkar, M., Busse, A., Sandham, N. D., "Direct numerical simulation of turbulent channel flow over a surrogate for Nikuradse-type roughness,” Journal of Fluid Mechanics, February 2018.

${ }^{11 .}$ Musker, A. J., “Universal Roughness Functions for Naturally-Occurring Surfaces,” Trans. Canadian Society for Mech. Eng., vol. 6, 1, 1980-81.

${ }^{12}$ Bons, J. P., “A Review of Surface Roughness Effects in Gas Turbines,” Journal of Turbomachinery, vol. 132, April 2010.

13.Forooghi, P., et al., “Toward a Universal Roughness Correlation,” Journal of Fluids Engineering, vol. 139, December 2017.

${ }^{14}$ Schultz, M. P., Flack, K. A., “Turbulent boundary layers on a systematically varied rough wall,” Phys. Fluids 21, January 2009.

${ }^{15 .}$ Napoli, E., Armenio, V., De Marchis, M., "The effect of the slope of irregularly distributed roughness elements on turbulent wall-bounded flows,” Journal of Fluid Mechanics, October 2008.

${ }^{16}$ Flack, K. A., Schultz, M. P., Barros, J. M., "Skin-friction behavior in the transitionally-rough regime," International Journal of Heat and Fluid Flow, June 2016.

${ }^{17}$ Barros, J. M., Flack, K. A., Schultz, M. P., “Measurements of skin-friction of systematically generated surface roughness,” International Journal of Heat and Fluid Flow, August 2018. 
${ }^{18 .}$ Modarress, D., Johnson, D. A., “Investigation of Turbulent Boundary-Layer Separation Using Laser Velocimetry,” AIAA Journal, Vol. 17, No. 7, 1979, pp. 747-752.

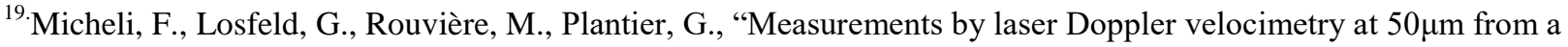
wall,” 9 $9^{\text {th }}$ French Congress of Velocimetry Laser, Bruxelles, Belgique, September 2004.

20.Clauser, F. H., “The Turbulent Boundary Layer,” Advances in Applied Mechanics, 4, 1956, pp. 1-51.

${ }^{21 .}$ Van Driest E.R., "Turbulent Boundary Layer in Compressible Fluids," Journal of the Aeronautical Sciences, Vol. 18, nº 3, 1951, pp. 145-160, 216.

${ }^{22 .}$ Cambier, L., Heib, S., Plot, S., "The ONERA elsA CFD Software: Input from Research and Feedback from Industry,” 28 ${ }^{\text {th }}$ ICAS Congress, Brisbane, 2012.

23.Jameson, A., Schmidt, W., and Turkel, E., "Numerical Solution of the Euler Equations by Finite Volume Methods Using Runge Kutta Time Stepping Schemes,” AIAA Paper 81-1259, June 1981.

${ }^{24 .}$ Menter, F. R., "Zonal Two Equation k- $\omega$ Turbulence Models for Aerodynamic Flows," AIAA Paper 93-2906, 1993.

${ }^{25 .}$ Aupoix, B., "Roughness Corrections for the k-x Shear Stress Transport Model: Status and Proposals,” Journal of Fluids Engineering, February 2015.

${ }^{26 .}$ Cartieri, A., Hue, D., Chanzy, Q., Atinault, O., "Experimental Investigations on Common Research Model at ONERA-S1MA-Drag Prediction Workshop Numerical Results,” Journal of Aircraft, Vol. 55, No. 4, JulyAugust 2018.

${ }^{27}$ Tinoco et al., "Summary Data from the Sixth AIAA CFD Drag Prediction Workshop: CRM Cases,” Journal of Aircraft, Vol. 55, No. 4, July-August 2018. 